\title{
Musgos (Bryophyta) de Bosques de PolyLePIS SERICEA (Rosaceae) del estado Mérida (Venezuela)
}

\author{
JESUS DELGADO ${ }^{1}$ y YELITZA LEÓN-VARGAS²
}

\begin{abstract}
Summary: The mosses (Bryophyta) of Polylepis sericea (Rosaceae) forests from Mérida state (Venezuela). Herein, we publish a list of 90 species of mosses associated with Polylepis sericea forests in Mérida, Venezuela. The Leucobryaceae family is the most diverse with 14 species followed by Bryaceae, Bartramiaceae and Pottiaceae. The most diverse genera are Campylopus (14 species), Leptodontium (5), Breutelia (4), Bryum (4), Zygodon (4) and Racomitrium (3). In these forests the acrocarpic mosses are dominant: represented by 68 species, of which tall turfs are the most frequent $(41.11 \%)$, followed by short turfs $(24.44 \%)$ and extensive mats $(8.89 \%)$. The phytogeographic analysis shows a predominance of neotropical elements (36\%), over worldwide distributed elements (27\%). Thirty-nine species are exclusive to Sierra Nevada of Mérida and 34 to Sierra de la Culata. Only 19\% of the species is shared by both Sierras, indicating that there is high beta diversity in the studied forests. In Venezuela the bryoflora of Polylepis forests is more similar to the Bolivian forests. The species Drepanocladus sordidus (Amblystegiaceae), Myurella sibirica (Plagiotheciaceae) and Pleurochaete luteola (Pottiaceae) are added to Venezuelan moss flora, as well as nine new records for the bryoflora of the Mérida state.
\end{abstract}

Key words: Bryophyta, forest, mosses, Paramo, Polylepis sericea, Venezuela.

Resumen: Se presenta un listado de 90 especies de musgos asociados a parches de bosques de Polylepis sericea Wedd., en el estado Mérida, Venezuela. La familia Leucobryaceae es la más diversa con 14 especies. Bryaceae, Bartramiaceae y Pottiaceae, le siguen en orden de importancia taxonómica. Los géneros más diversos son Campylopus (14 especies), Leptodontium (5), Breutelia (4), Bryum (4), Zygodon (4) y Racomitrium (3). La condición acrocárpica es la dominante, representada por 68 especies, siendo los tepes altos los más frecuentes $(41,11 \%)$, seguidos por los tepes cortos $(24,44 \%)$ y los tapetes extensivos $(8,89 \%)$. El análisis fitogeográfico muestra una predominancia del elemento neotropical (36\%) sobre el elemento ampliamente distribuido en el mundo (27\%). En la Sierra Nevada de Mérida, son exclusivas 39 especies y 34 en la Sierra de La Culata. Solo el 19\% de las especies es compartido por ambas sierras, indicando que existe un recambio de las especies en los parches de bosque inventarios lo que podría estar asociado con variables físicas y ambientales de cada localidad. Se adicionan las especies Drepanocladus sordidus (Amblystegiaceae), Myurella sibirica (Plagiotheciaceae) y Pleurochaete luteola (Pottiaceae) a la brioflora venezolana, así como nueve nuevos registros para la brioflora del estado Mérida.

Palabras clave: Bosques, Bryophyta, musgos, páramo, Polylepis sericea, Venezuela.

\section{INTRODUCCIÓN}

Los bosques altoandinos de Polylepis Ruiz \& Pav., son sistemas forestales complejos, no sólo por

\footnotetext{
1 Postgrado en Botánica Taxonómica Neotropica (BOTANE), Instituto Jardín Botánico de Mérida. Facultad de Ciencias. Universidad de Los Andes, Mérida, Venezuela.

${ }^{2}$ Instituto Jardín Botánico de Mérida. Facultad de Ciencias. Universidad de Los Andes, Mérida, Venezuela.
}

los roles ecológicos que cumplen en la dinámica en la alta montaña tropical y subtropical, sino también por los factores antropogénicos que están implicados en su distribución (Gareca et al. 2010; Toivonen, 2014). Polylepis, está constituido por especies en su mayoría arbóreas, que se distribuyen en los bosques montanos y altoandinos de la Cordillera de Los Andes, desde Venezuela hasta el norte de Chile y el noreste-centro de Argentina (Simpson, 1979; Kessler, 2002; Mendoza \& Cano, 2011). Este género incluye especies de árboles o 
arbustos, que forman bosques con poblaciones muy fragmentadas en toda la extensión de la Cordillera de Los Andes (Kessler \& Schmidt-Lebuhn, 2006; Mendoza, 2010; Mendoza \& Cano, 2011; Zutta et al. 2012).

Los bosques de coloradito ("queñoa", "queñua" o "queuña”, según el país) actúan como protectores de las porciones superiores de cuentas fluviales, constituyendo una fuente importante de agua al aumentar la capacidad de penetración de ésta en el suelo, regulando la escorrentía y controlando los procesos erosivos (Renison \& Cingolani, 1998). Además, en estos bosques se encuentran poblaciones de aves y pequeños mamíferos que están amenazadas por el uso y reducción del hábitat, razón por la cual figuran como recursos biológicos de alta fragilidad y relevancia.

Son muy importantes en la dinámica de los procesos ecosistémicos en el páramo debido a su ubicación sobre la línea del timberline, línea alcanzada por especies arbóreas de los géneros Escallonia, Libanothamnus, Diplostephium y Oreopanax. A lo largo de este cambio gradual desde vegetación arbórea hasta llegar a formas arbustivas y finalmente a vegetación paramera (Körner \& Paulsen, 2004; Toivonen, 2014), los bosques de Polylepis llegan a distribuirse como islas dentro de la vegetación de páramo, resultando así, en uno de los ecosistemas de esta región más complejos y difíciles de interpretar (Monasterio, 1980a, b).

En cuanto a su distribución, estos bosques se disponen de manera fragmentaria entre los (2600) 3500 y 4900 m. Los bosques de mayor altitud, han sido reportados por Hoch \& Körner (2005) en el volcán Sajama, Bolivia a 4810 m, mientras que Marcora et al. (2008) reportan parches de bosques de Polylepis a $800 \mathrm{~m}$ en las montañas de Córdoba, Argentina. El origen del ordenamiento espacial de los bosques actuales se ha atribuido a eventos asociados a condiciones fisiológicas y microambientales particulares (Simpson, 1986), aunque se ha propuesto que su distribución es de origen relictual, producto de la fragmentación de un amplio cinturón de Polylepis que ocupó los Andes durante el Pleistoceno (Hueck, 1962).

Actualmente, los bosques de Polylepis spp., son considerados como uno de los ecosistemas más amenazados de América (Navarro et al. 2005), hecho que está asociado con el estado de transformación y fragmentación de estos enclaves por acción antrópica, por lo que su conocimiento y protección son una tarea urgente.

Los musgos son plantas de amplia distribución que crecen más favorablemente en regiones húmedas y sombreadas de baja evapotranspiración. Son de vital importancia debido a su rol como interceptores de lluvia ya que adsorben agua y la retienen, contribuyendo a evitar la erosión de los suelos (Churchill \& Linares, 1995). En Los Andes, los bosques de Polylepis con microambientes muy húmedos, particularmente aquellos formados por Polylepis sericea Wedd., P. pauta Hieron, P. pepei B.B. Simpson y P. lanata (Kuntze) M. Kessler \& Schmidt-Leb., pueden llegar a albergar una buena cobertura epífita donde las briofitas, bromelias y algunos helechos son dominantes (Kessler, 2006; Aldana, 2008). En estos bosques las briofitas terrícolas y saxícolas son elementos comunes que pueden formar un tapiz continuo en el suelo ayudando así al mantenimiento de la humedad.

Pocos son los estudios que incluyen a las briofitas en bosques de Polylepis. En Colombia, Wolf (1993) describe las comunidades de criptógamas epífitas en un bosque de Polylepis sericea por encima de los $4000 \mathrm{~m}$. Según este autor, las briofitas Daltonia longifolia Taylor, Blepharolejeunea securifolia (Steph.) R.M. Schust. y Frullania tetraptera Nees \& Mont. son especies frecuentes, así como Campylopus pittieri R.S. Williams, C. fragilis (Brid.) Bruch \& Schimp., Chorisodontium mittenii (Müll. Hal.) Broth., Leptodontium wallisii (Müll. Hal.) Kindb., Porotrichodendron superbum (Taylor) Broth. y Zygodon fragilis $\mathrm{H}$. Rob. Este autor reporta 14 especies de musgos y 13 especies de hepáticas para este bosque.

Por su parte, Parolly \& Kürschner (2005) indican que en bosques de Polylepis ecuatorianos, las especies de briofitas se distribuyen en función de parámetros ecológicos asociados a humedad, y describen dos asociaciones vegetales; la primera dominada por Blepharolejeunea securifolia (Stephani) R. M. Schust y Orthotrichum trachymitrium Mitt, especies frecuentes en zonas secas junto a Cryphaea ramosa (Mitt.) Wilson, Orthotrichum pycnophyllum Schimp., Streptopogon erythrodontus (Taylor) Wilson, Frullania dusenii Steph., F. ecklonii (Spreng.) Gottsche, Lindenb. \& Nees, Cryphaea patens 


\section{J. Delgado y Y. León - Musgos de los bosques de Polylepis en Venezuela}

Hornsch. ex Müll. Hal. y Thuidium peruvianum Mitt.; mientras que en las zonas con mayor humedad dominan los musgos Campylopus albidovirens Herzog y Lepyrodon tomentosus (Hook.) Mitt., junto a Adelanthus decipiens (Hook.) Mitt., Brachythecium cirriphylloides K.D. McFarland, Campylopus areodictyon (Müll. Hal.) Mitt., Cephaloziella granatensis, (J.B. Jack ex Steph.) Fulford, Chorisodontium wallisii (Müll. Hal.) Broth. y Daltonia tenuifolia Mitt. De manera general, sus listados incluyen unas 45 especies de musgos y 30 especies de hepáticas asociadas a estos enclaves forestales.

En Bolivia, Aldana (2008) describe 19 comunidades de briofitas en bosques de Polylepis pepei B.B. Simpson., diferenciando dos comunidades generalistas de roca dominadas por Campylopus areodictyon (Müll. Hal.) Mitt. y Racomitrium crispipilum (Taylor) A. Jaeger), seis en rocas bajo parches de Polylepis, dominadas por las especies Braunia rupestris (Mitt.) A. Jaeger, Leptodontium tricolor (R.S. Williams) R.H. Zander, Campylopus nivalis (Brid.) Brid. var. nivalis J. P. Frahm., Breutelia chrysea (Müll. Hal.) A. Jaeger, Bryum leptotorquescens Müll. Hal. ex Broth. e Hypnum amabile (Mitt.) Hampe, cuatro comunidades en rocas de sitios abiertos con Andreaea spp. y Campylopus spp., cinco sobre troncos de Polylepis (con Frullania spp, Rhodobryum grandifolium (Taylor) Schimp. y Symblepharis lindigii Hampe) y dos en suelo de sitios abiertos dominados por Oreoweisia erosa (Hampe ex Müll. Hal.) Kindb. y Chrysoblastella chilensis (Mont.) Reimers. Esta autora lista un total de 83 especies de musgos y 37 especies de hepáticas para los bosques inventariados.

En Venezuela, los bosques de Polylepis son considerados "Zonas de Protección Integral", junto a los humedales y turberas de montaña. Sin embargo, el conocimiento en función de la diversidad asociada a éstos dista de estar completo. Este trabajo es el primer aporte al conocimiento de la flora muscícola en bosques de Polylepis para el país. Con este fin, se presenta un primer listado de musgos, con nuevas adiciones a la flora briofítica venezolana y se hace un análisis por preferencias de sustratos y formas de vida así como un análisis fitogeográfico de las especies. Se establecen afinidades florísticas entre cinco parches de bosques de Polylepis en la cordillera de Mérida.

\section{Materiales Y MÉtodos}

\section{Área de estudio}

Los Andes venezolanos están constituidos por grandes núcleos de páramo separados por valles tectónicos profundos que dan origen a las diferentes sierras (Monasterio \& Reyes, 1980), que en conjunto forman a la Cordillera de Mérida. Al Sur se ubica la Sierra Nevada de Mérida, que es la parte del Ramal mejor conocida, y que alberga pequeños glaciares y las cumbres más altas de Venezuela $(5007 \mathrm{~m}$, Pico Bolívar). Al norte se ubica la Sierra de la Culata que está caracterizada por formar un valle casi recto en toda su extensión. De manera general, el área de distribución de los bosques de Polylepis en ambas sierras de la Cordillera, está representada por vertientes abruptas con desniveles, que se deben a la influencia de quebradas que fluyen a lo largo de valles y complejos morrénicos (Schubert \& Valastro, 1973). En esta Cordillera, los bosques de Polylepis ocupan un área aproximada de 205.000 hectáreas, siendo la Sierra Nevada de Mérida la que alberga mayor cantidad de superficie boscosa en comparación con la de la Culata (Arnal, 1983).

\section{Selección de los bosques}

Se seleccionaron cinco parches de bosques de Polylepis, cuatro en la Sierra Nevada de Mérida con un área aproximada de una hectárea cada uno, y otro de aproximadamente 3 hectáreas en la Sierra de La Culata. Todos los bosques se ubican en afloramientos o derrubios rocosos fisurados a más de $3000 \mathrm{~m}$, cota altitudinal que define a la vegetación de páramo. La ubicación local de los bosques se muestra en la Tabla 1, y su ubicación geográfica relativa junto a una vista general de los mismos en la Figura 1.

\section{Vegetación}

El estrato superior está dominado por Polylepis sericea (coloradito) de tronco rojizo y retorcido con capas de corteza que se desprenden, acompañado por árboles del género Gynoxys (León, 1991) o Libanothamnus (Delgado, 2015). En el sotobosque, ocupando una posición intermedia, se encuentran especies de los géneros Valeriana, Senecio, Gnaphalium, Berberis y Ribes. Postradas en ese mismo estrato, la familia Ericaceae con los géneros Gaultheria, Vaccinum y Disterigma, está muy bien representada. Las ramas de los árboles y arbustos 
Tabla 1. Localización geográfica de los bosques de Polylepis en el estado Mérida, Venezuela.

\begin{tabular}{|c|c|c|c|c|}
\hline Bosque & Localidad & Coordenadas & $\begin{array}{l}\text { Altitud } \\
\text { (m) }\end{array}$ & $\begin{array}{l}\text { Superficie } \\
\text { (ha) }\end{array}$ \\
\hline Bosque 1 & $\begin{array}{l}\text { Estación Loma Redonda, Sistema teleférico de Mérida, Sierra } \\
\text { Nevada, VENEZUELA. Bosque en las adyacencias de la estación. }\end{array}$ & $\begin{array}{l}8^{\circ} 32^{\prime} 44,50 " \mathrm{~N} \\
71^{\circ} 04^{\prime} 49,87^{\prime \prime} \mathrm{W}\end{array}$ & 3953 & 1.2 \\
\hline Bosque 2 & $\begin{array}{l}\text { Estación Loma Redonda, Sistema teleférico de } \\
\text { Mérida, Sierra Nevada, VENEZUELA. Bosque } \\
\text { colindando con la Laguna Los Anteojos. }\end{array}$ & $\begin{array}{l}8^{\circ} 32^{\prime} 08,53^{\prime \prime} \mathrm{N} \\
71^{\circ} 04^{\prime} 28,91^{\prime \prime} \mathrm{W}\end{array}$ & 4011 & 1.1 \\
\hline Bosque 3 & $\begin{array}{l}\text { Estación Loma Redonda, Sistema teleférico de Mérida, } \\
\text { Sierra Nevada, VENEZUELA. Bosque en el camino al } \\
\text { "Alto de la Cruz" - camino al pueblo "Los Nevados". }\end{array}$ & $\begin{array}{l}8^{\circ} 32^{\prime} 11,33 " \mathrm{~N} \\
71^{\circ} 04^{\prime} 44,06, \mathrm{~W}\end{array}$ & 4122 & 0.93 \\
\hline Bosque 4 & $\begin{array}{l}\text { Páramo El Escorial, Sierra de La Culata, Estado Mérida, } \\
\text { VENEZUELA. Bosque adyacente a la Laguna "La Barrosa". }\end{array}$ & $\begin{array}{l}8^{\circ} 44^{\prime} 34,30^{\prime \prime} \mathrm{N} \\
71^{\circ} 02^{\prime} 45,31^{\prime \prime} \mathrm{W}\end{array}$ & 3618 & 3.1 \\
\hline Bosque $5^{*}$ & $\begin{array}{l}\text { Páramo de Mucubají, Valle del Mucubají, Sierra } \\
\text { Nevada, VENEZUELA. Bosque que da fin al Valle. }\end{array}$ & $\begin{array}{l}8^{\circ} 46 ' 46,40 " \mathrm{~N} \\
70^{\circ} 49^{\prime} 14,98 \text { ' } \mathrm{W}\end{array}$ & 3692 & 1.1 \\
\hline
\end{tabular}

*Los datos del inventario en este bosque, se corresponde únicamente con el borde del mismo (datos tomados de Polanco, 2015).

alojan epifitos como helechos de hojas delgadas de la familia Hymenophyllaceae junto con especies de Peperomia, musgos y hepáticas. Plantas de pequeño porte de los géneros Geranium, Bartsia y Oxalis, conforman el estrato herbáceo del bosque, junto a especies tapizantes de Lachemilla. En los claros, se encuentran plantas de páramo abierto de los géneros Espeletia, Coespeletia, Hypericum, Sisyrrhynchium, Orthrosanthus y Castilleja así como diferentes especies de gramíneas.
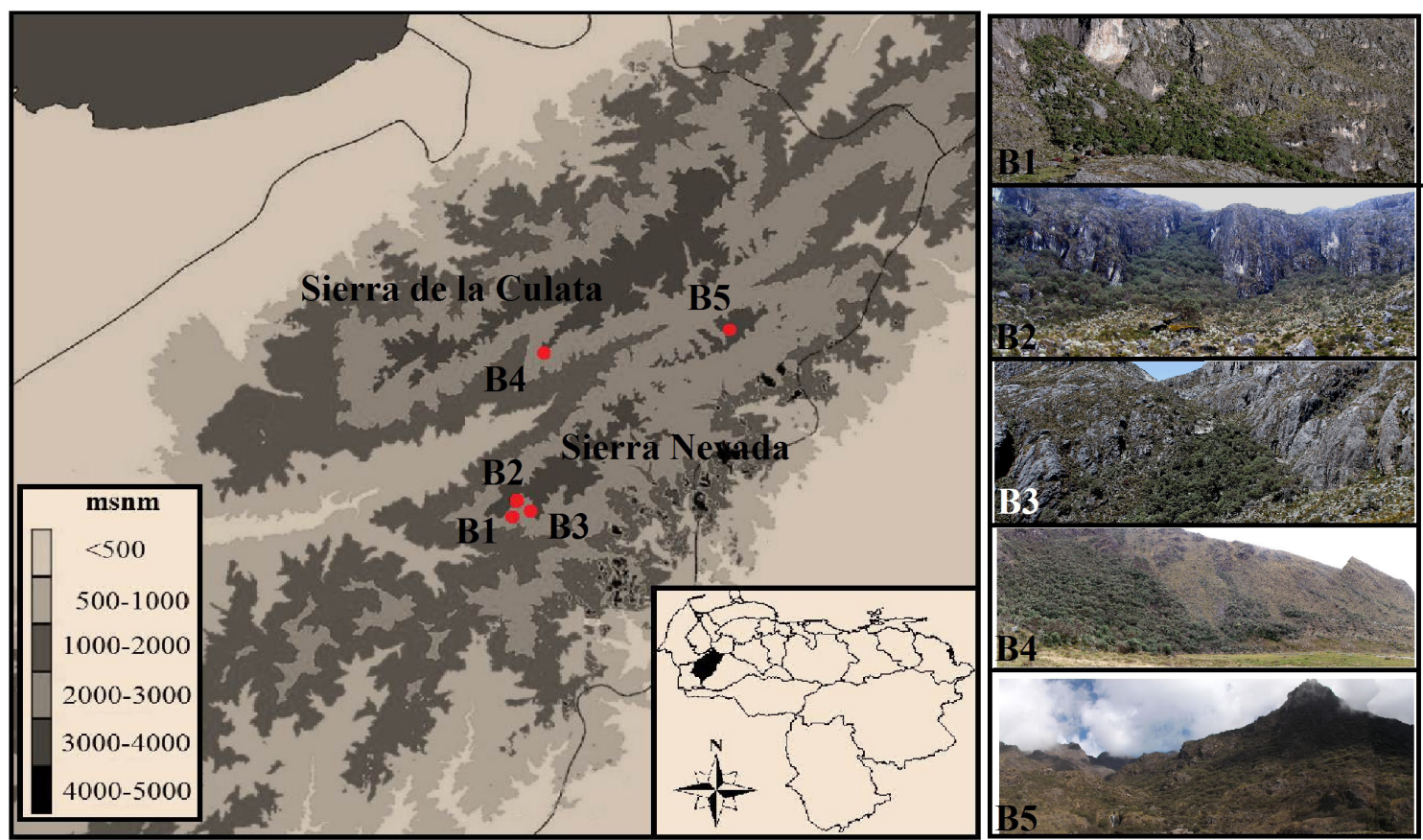

Fig. 1. Ubicación geográfica relativa de los parches de bosques de Polylepis inventariados y vista general de los mismos. 
Análisis florístico: colecta y determinación del material vegetal

Se colectaron muestras de briofitas siguiendo el protocolo de Frahm (2003). Las colectas fueron realizadas durante los años 2013 y 2015. Al inventario, se le adicionaron los especímenes depositados en MERC, Colección de Criptógamas del Instituto Jardín Botánico de Mérida, Venezuela colectados por León-Vargas en los bosques de la Sierra Nevada de Mérida.

Para las determinaciones se utilizaron las claves de Churchill \& Linares (1995) y Gradstein et al. (2001), así como las de la base de datos pública para briofitas de Los Andes Tropicales (http:// www.tropicos.org/Project/ANBRY, Missouri Botanical Garden). Se emplearon las claves de Crum \& Anderson (1981) de los musgos de Norteamérica y Sharp et al. (1994) de los musgos de México. Además, se utilizaron claves para grupos particulares: Sphagnum (Griffin, 1981), Campylopus (Frahm, 1991), Zygodon (Griffin, 1990), Orthotrichum (Lewinsky, 1993), Grimmia (Muñoz, 1999), Pottiaceae (Zander, 1993), Amblystegiaceae (Hedenäs, 2003), Bartramiaceae (Fransen, 2005) y Bryaceae (Ochi, 1980, 1981). Las especies determinadas fueron comparadas con muestras en el Herbario MERC del Instituto Jardín Botánico de Mérida y fueron depositadas en este mismo Herbario.

Para el listado se sigue el criterio taxonómico para las familias según Goffinet \& Shaw (2009) disponible en http://bryology.uconn.edu/ classification-2, y para géneros y especies los de Churchill \& Linares (1995). En el listado de especies encontradas en los parches inventariados se indican forma de crecimiento, forma de vida, distribución mundial y por sierras en la Cordillera. Los datos asociados a las especies se encuentran en la Base de datos Musgos de Venezuela (MdV) de León et al. (2014) (disponible en http:// musgos.cecalc.ula.ve/)

\section{Análisis fitogeográfico}

Para el análisis fitogeográfico se hicieron algunas modificaciones de los criterios establecidos por Van Der Wijk et al. (1959) siguiendo lo propuesto por Schofield (1992) sobre los reinos brioflorísticos del mundo. La nomenclatura utilizada es la empleada por Van Der Wijk (1959) en el mapa de distribución fitogeográfica de musgos del Index Muscorum, el cual divide al mundo en regiones brioflorísticas: Elemento Neotropical (EN), aquellas especies distribuidas en las regiones Am 2 - 5 (en más de dos Am); Elemento Andino (EA), especies confinadas a los Andes Tropicales; Elemento ampliamente distribuido en América $(\operatorname{AdA})$, especies distribuidas en Am $1-6$ (en más de una región); Elemento Pantropical (P), especies distribuidas tanto en América del Sur como en África Subsahariana y parte de Asia meridional; Elemento ampliamente distribuido en el mundo (AdM) especies distribuidas en Am, Afr, As, Austr, Eur, Oc (en más de un continente) y Cosmopolitas (C), especies distribuidas en todas las regiones brioflorísticas.

\section{Similaridad florística}

La similaridad florística entre los parches de bosques de Polylepis inventariados fue medida en función de la presencia/ausencia de las especies en cada parche. Se estimó el índice de similitud de Sørensen y se construyó un cluster en función de las relaciones existentes en términos de composición florística usando PAST (Hammer et al. 2001), un software de acceso libre (disponible en http://nhm2. uio.no/norlex/past/download.html). Se compara la diversidad florística de los parches de Polylepis con los inventarios hechos por Wolf (1993) en Colombia, Parolly \& Kürschner (2005) en Ecuador y Aldana (2008) en Bolivia, sólo en función de la presencia/ausencia de las especies.

\section{Resultados}

\section{Composición florística}

Se listan un total de 90 especies de musgos, distribuidos en 53 géneros y 29 familias (Tabla 2). El análisis general por categorías taxonómicas indica que la familia mejor representada es Leucobryaceae, con una riqueza específica de 14 especies, todas del género Campylopus. Bryaceae, Bartramiaceae y Pottiaceae, son las familias que le siguen, cada una con 9 especies, mientras que Polytrichaceae y Orthotrichaceae figuran con 6 especies cada una. Del total de familias de Bryophyta, 15 están representadas por un único género y una especie, lo cual podría ser un indicador de la heterogeneidad florística en los bosques (Figura 2). 


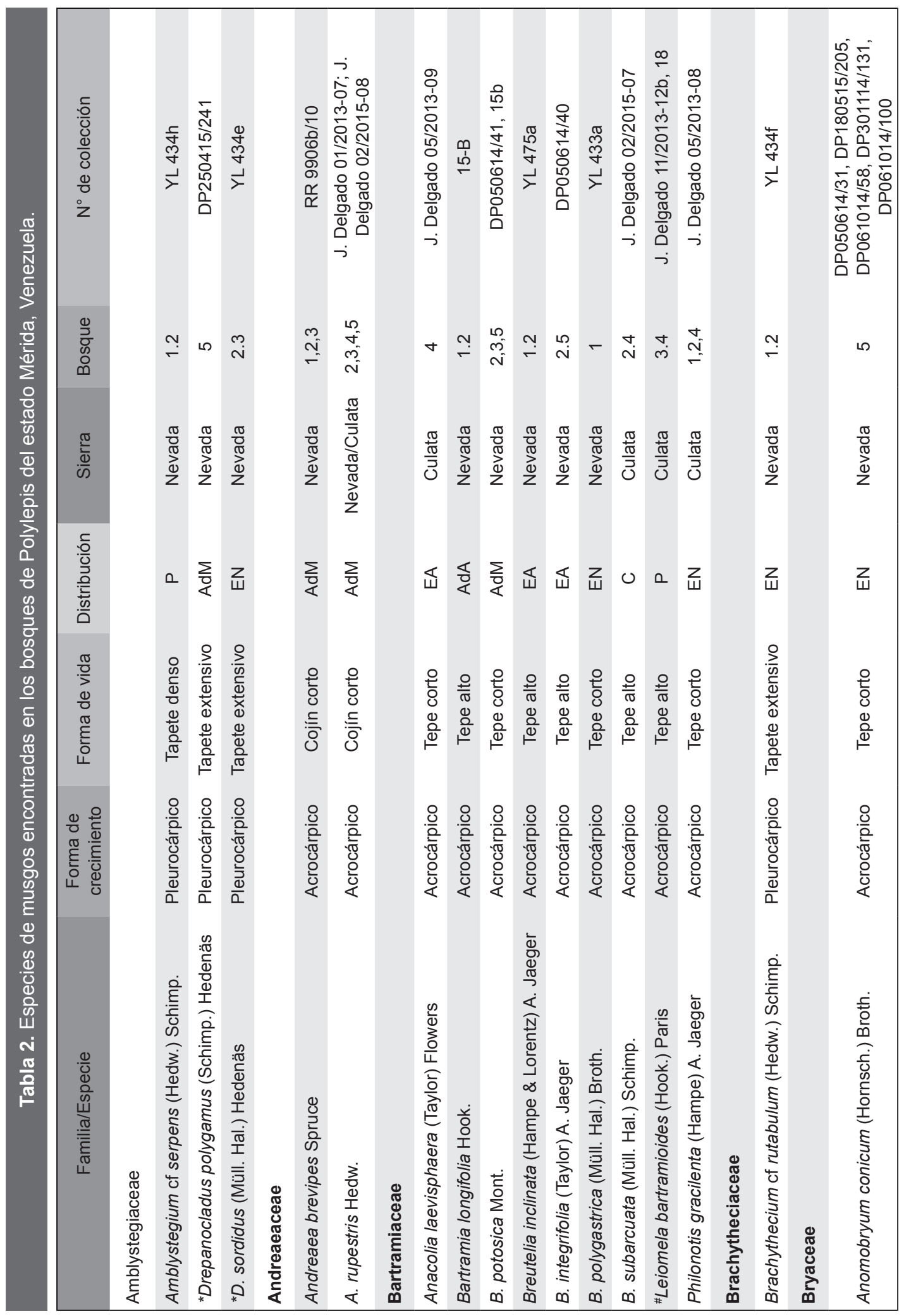




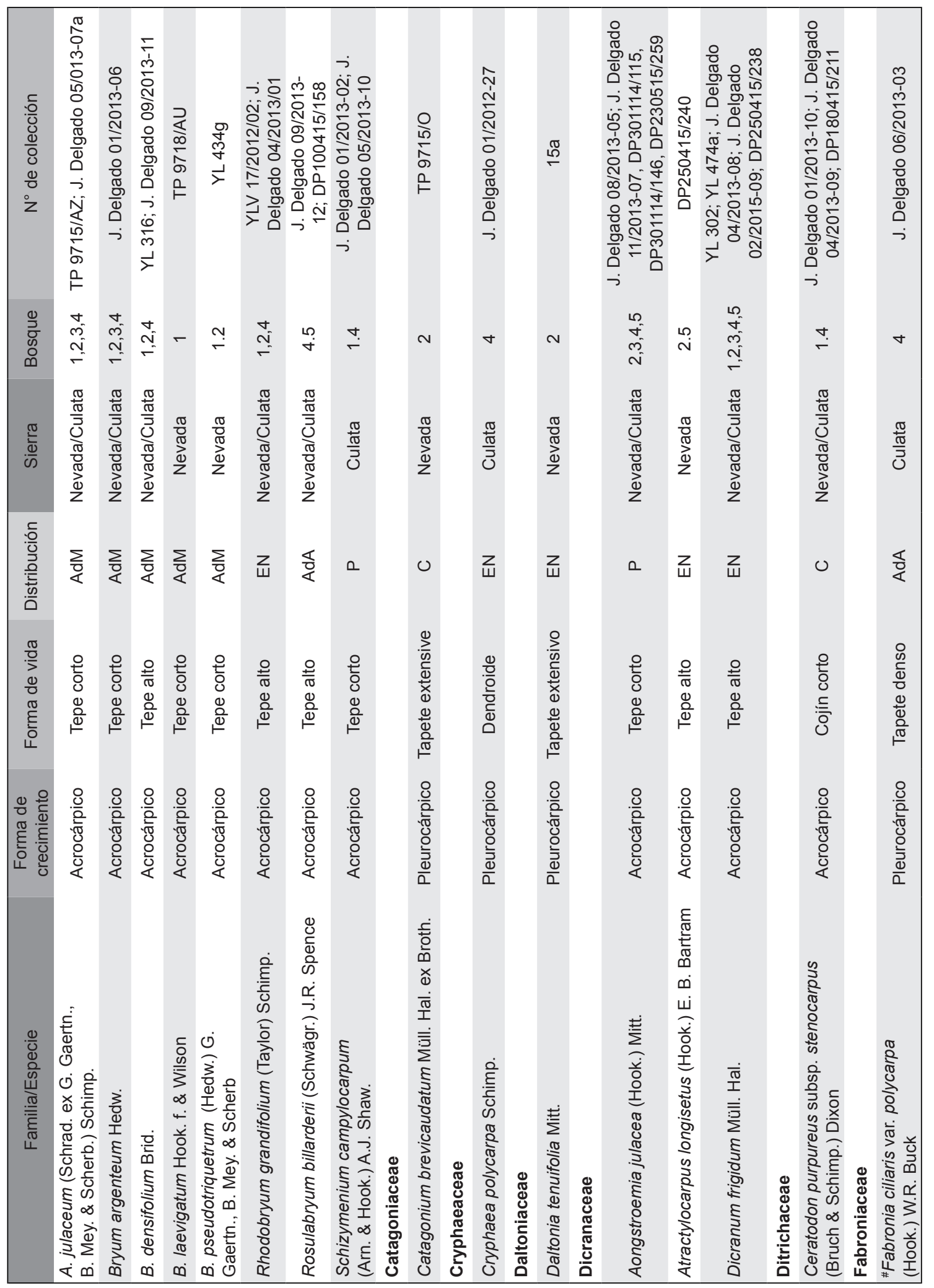




\begin{tabular}{|c|c|c|c|c|c|c|c|c|c|c|c|c|c|c|c|c|}
\hline 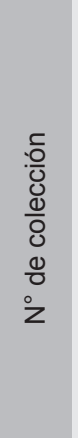 & & 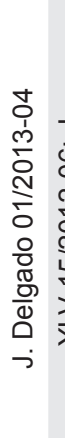 & 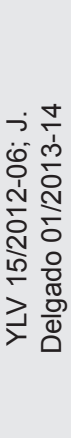 & 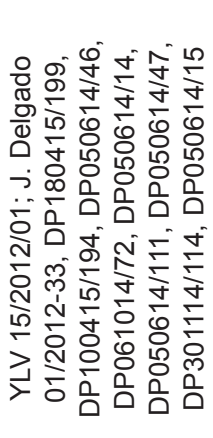 & 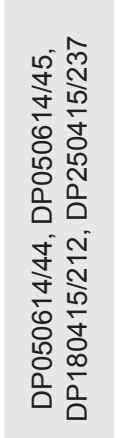 & 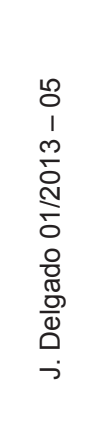 & & 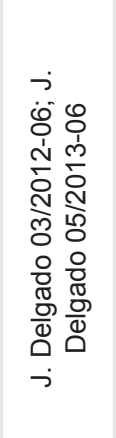 & 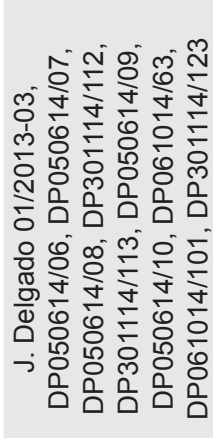 & & 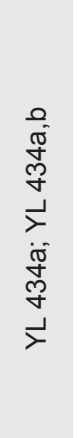 & 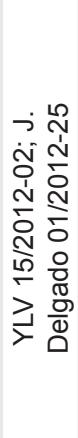 & & 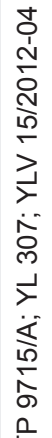 & & $\begin{array}{l}3 \\
\vdots \\
\infty \\
\vdots \\
0 \\
0 \\
1 \\
1\end{array}$ \\
\hline 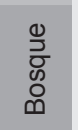 & & $\begin{array}{l}\stackrel{+}{c} \\
\stackrel{2}{\sim} \\
\stackrel{-}{-}\end{array}$ & $\stackrel{\grave{i}}{\sim}$ & $\begin{array}{l}\stackrel{0}{0} \\
\stackrel{5}{-} \\
\stackrel{5}{i} \\
\stackrel{-}{\leftarrow}\end{array}$ & ما & $\begin{array}{l}\stackrel{+}{\mathrm{s}} \\
\stackrel{\mathrm{N}}{ }\end{array}$ & & $\nabla$ & $\begin{array}{l}n \\
+ \\
\tilde{\infty} \\
\stackrel{-}{-}\end{array}$ & & - & $\begin{array}{l}\stackrel{+}{\sim} \\
\stackrel{-}{\sim} \\
\stackrel{-}{r}\end{array}$ & & $\begin{array}{l}\stackrel{+}{\sigma} \\
\stackrel{n}{N} \\
\stackrel{2}{\sigma}\end{array}$ & & $\stackrel{m}{\leftarrow}$ \\
\hline$\frac{\pi}{\frac{\pi}{\omega}}$ & & $\begin{array}{l}\frac{\pi}{\pi} \\
\frac{\pi}{J} \\
0\end{array}$ & $\frac{\frac{\pi}{\pi}}{\frac{\pi}{J}}$ & $\begin{array}{l}\frac{\pi}{0} \\
\frac{\pi}{0} \\
\stackrel{0}{2}\end{array}$ & $\begin{array}{l}\frac{\pi}{0} \\
\frac{\pi}{0} \\
\frac{0}{2}\end{array}$ & $\frac{\frac{\pi}{\pi}}{\frac{\pi}{5}}$ & & $\frac{\frac{\pi}{\pi}}{\frac{\pi}{5}}$ & $\begin{array}{l}\frac{\pi}{J} \\
\frac{0}{0} \\
\frac{\pi}{0} \\
\frac{\pi}{\pi} \\
\frac{d}{2}\end{array}$ & & $\begin{array}{l}\frac{\pi}{0} \\
\frac{\pi}{\pi} \\
\frac{0}{2}\end{array}$ & $\frac{\frac{\pi}{\pi}}{\frac{\pi}{5}}$ & & $\frac{\frac{\pi}{\pi}}{\frac{\pi}{5}}$ & & $\begin{array}{l}\frac{\pi}{0} \\
\frac{\pi}{2} \\
\frac{\mathbb{d}}{2}\end{array}$ \\
\hline 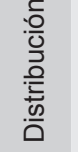 & & $\sum_{0}$ & 凹 & $\sum_{\substack{\alpha\\
}}$ & $\sum_{\substack{\alpha \\
<}}$ & 0 & & $\sum_{\substack{\alpha \\
<}}$ & $\sum_{\substack{\alpha \\
<}}$ & & Z & Z & & $\underset{\mathbb{2}}{\mathbb{2}}$ & & 凹 \\
\hline 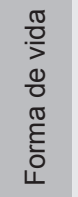 & & 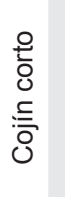 & $\begin{array}{l}\text { 웜 } \\
\text { ơ } \\
: \frac{\bar{\sigma}}{0}\end{array}$ & 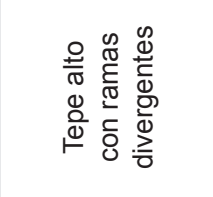 & 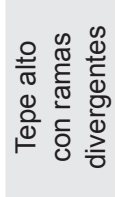 & 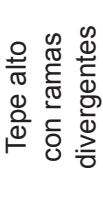 & & 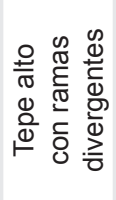 & 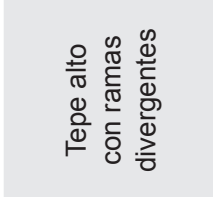 & & 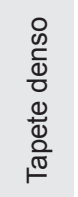 & 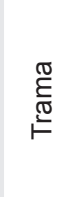 & & 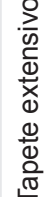 & & 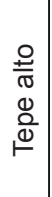 \\
\hline 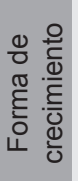 & & $\begin{array}{l}\frac{0}{2} \\
\frac{0}{0} \\
\frac{0}{0} \\
\frac{0}{4}\end{array}$ & $\begin{array}{l}0 \\
\frac{0}{0} \\
\frac{0}{07} \\
\frac{0}{0} \\
\frac{0}{0} \\
\frac{0}{4}\end{array}$ & $\begin{array}{l}\frac{0}{2} \\
\frac{0}{20} \\
\frac{10}{0} \\
\frac{0}{0} \\
\frac{0}{4}\end{array}$ & 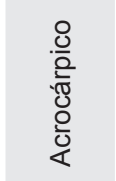 & 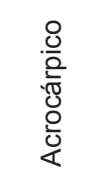 & & $\begin{array}{l}\frac{0}{0} \\
\frac{0}{20} \\
\frac{10}{0} \\
\frac{0}{0} \\
\frac{0}{4}\end{array}$ & $\begin{array}{l}\frac{0}{0} \\
\frac{0}{0} \\
\frac{\pi}{0} \\
\frac{0}{0} \\
\frac{0}{4}\end{array}$ & & $\begin{array}{l}\frac{8}{0} \\
\frac{.0}{0.0} \\
\frac{0}{3} \\
\frac{0}{0}\end{array}$ & 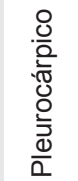 & & $\begin{array}{l}.0 \\
. \frac{0}{2} \\
\frac{\pi}{\pi} \\
\frac{0}{3} \\
\frac{0}{0}\end{array}$ & & $\begin{array}{l}\frac{8}{2} \\
. \frac{0}{0} \\
\frac{0}{0} \\
\frac{0}{0} \\
\frac{0}{4}\end{array}$ \\
\hline 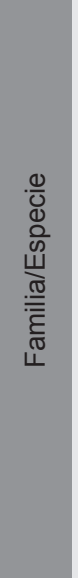 & 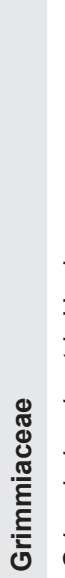 & 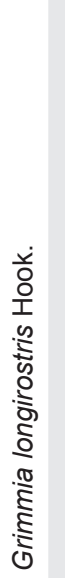 & 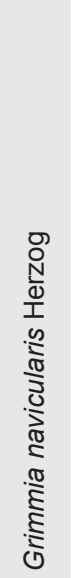 & 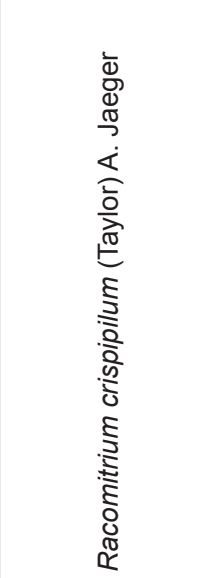 & 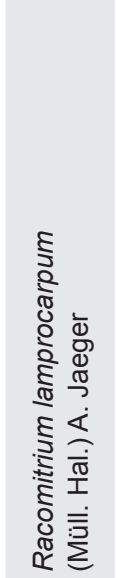 & 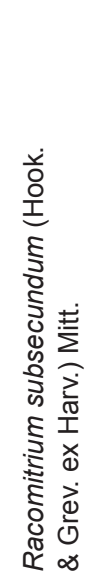 & 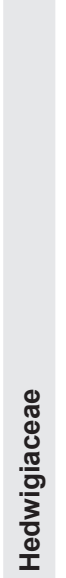 & 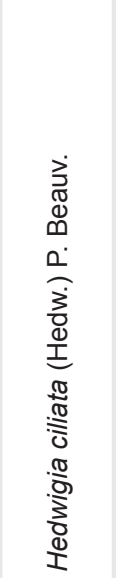 & 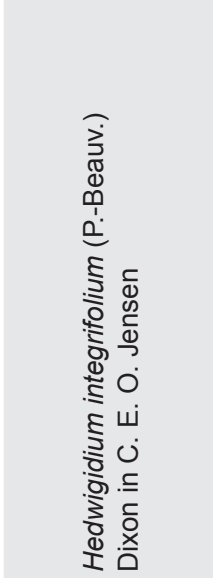 & 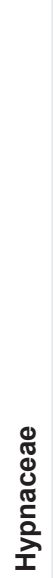 & 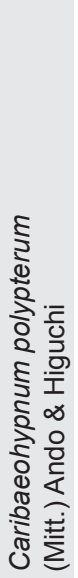 & 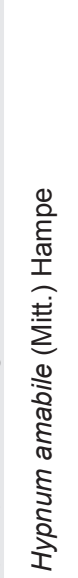 & 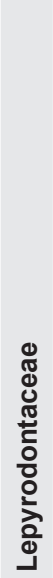 & 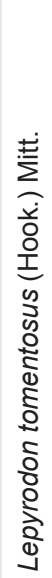 & 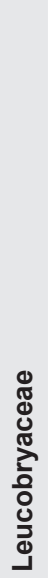 & 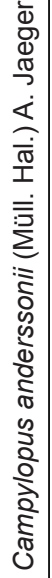 \\
\hline
\end{tabular}




\begin{tabular}{|c|c|c|c|c|c|c|c|c|c|c|c|c|c|c|c|c|c|c|c|c|}
\hline 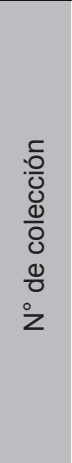 & 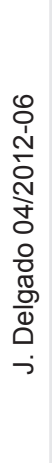 & 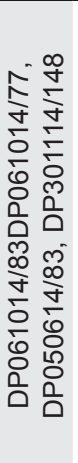 & 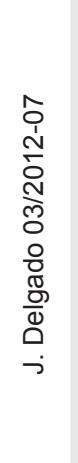 & 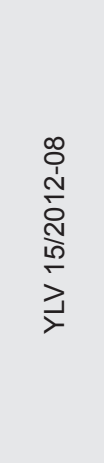 & 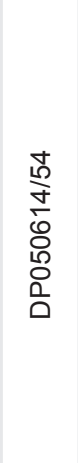 & 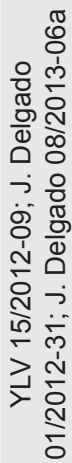 & 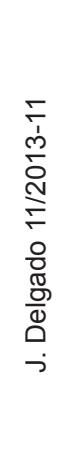 & 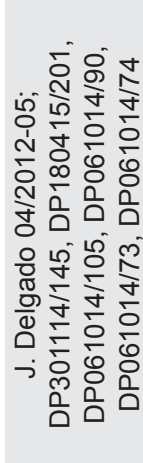 & $\begin{array}{l}\stackrel{\bar{m}}{\nu} \\
\dot{\nu} \\
\dot{\theta} \\
\stackrel{\infty}{\nu} \\
\stackrel{\nu}{\nu}\end{array}$ & ż & 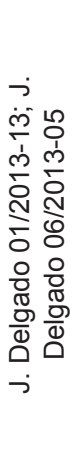 & 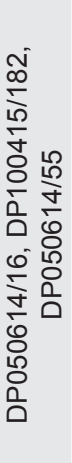 & $\frac{z}{\omega}$ & & 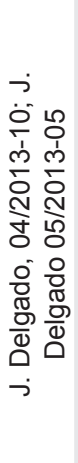 & 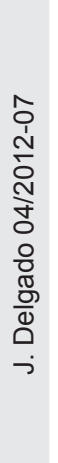 & 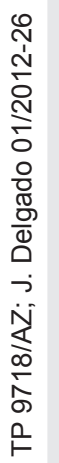 & & 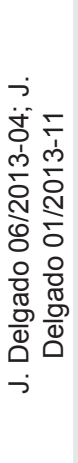 & 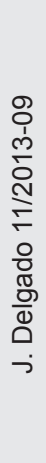 \\
\hline $\begin{array}{l}0 \\
\frac{0}{0} \\
0 \\
0 \\
\infty\end{array}$ & $\begin{array}{l}\stackrel{+}{\sim} \\
\stackrel{-}{-}\end{array}$ & م & $\nabla$ & $\begin{array}{l}\stackrel{+}{m} \\
\stackrel{\sim}{-}\end{array}$ & L & $\begin{array}{l}\stackrel{0}{0} \\
\stackrel{-}{N} \\
\stackrel{-}{\leftarrow}\end{array}$ & $\nabla$ & $\begin{array}{l}\stackrel{+}{\text { m}} \\
\stackrel{N}{*}\end{array}$ & - & - & $\begin{array}{l}\stackrel{+}{m} \\
\stackrel{n}{\sim} \\
\stackrel{-}{2}\end{array}$ & م & $\stackrel{m}{\longrightarrow}$ & & 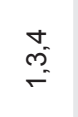 & $\nabla$ & 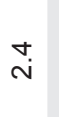 & & $\nabla$ & $\nabla$ \\
\hline$\frac{\pi}{\frac{\pi}{\omega}}$ & $\frac{\frac{\pi}{\pi}}{\frac{\pi}{J}}$ & $\begin{array}{l}\frac{\pi}{2} \\
\frac{\pi}{2} \\
\frac{0}{2}\end{array}$ & $\begin{array}{l}\frac{\pi}{\pi} \\
\frac{\pi}{5}\end{array}$ & $\frac{\frac{\pi}{\pi}}{\frac{\pi}{5}}$ & $\begin{array}{l}\frac{\pi}{0} \\
\frac{\pi}{2} \\
\frac{0}{2}\end{array}$ & $\begin{array}{l}\frac{\pi}{0} \\
\frac{\pi}{2} \\
0 \\
0 \\
\frac{\pi}{0} \\
\frac{\pi}{0} \\
\frac{0}{2} \\
Z\end{array}$ & $\frac{\frac{\pi}{\pi}}{\frac{\pi}{J}}$ & $\begin{array}{l}\frac{\pi}{\pi} \\
\frac{\pi}{5} \\
0 \\
\frac{\pi}{\pi} \\
\frac{\pi}{\pi} \\
\frac{\pi}{2} \\
z\end{array}$ & $\begin{array}{l}\frac{\pi}{2} \\
\frac{\pi}{2} \\
2 \\
2\end{array}$ & $\begin{array}{l}\frac{\pi}{2} \\
\frac{\pi}{2} \\
2 \\
2\end{array}$ & $\frac{\frac{\pi}{\pi}}{\frac{\pi}{J}}$ & $\begin{array}{l}\frac{\pi}{2} \\
\frac{\pi}{2} \\
2 \\
2\end{array}$ & $\begin{array}{l}\frac{\pi}{0} \\
\text { 20 } \\
\text { d }\end{array}$ & & $\frac{\frac{\pi}{\pi}}{\frac{\pi}{5}}$ & $\frac{\frac{\pi}{\pi}}{\frac{\pi}{J}}$ & $\begin{array}{l}\frac{\pi}{\pi} \\
\frac{\pi}{5} \\
\frac{0}{\pi} \\
\frac{\pi}{0} \\
\frac{\pi}{0} \\
\frac{d}{Z}\end{array}$ & & $\frac{\frac{\pi}{\pi}}{\frac{\pi}{J}}$ & $\frac{\frac{\pi}{0}}{\frac{\pi}{5}}$ \\
\hline 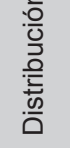 & ㄴ & 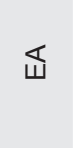 & $\sum_{0}$ & 0 & 岀 & ㄴ & 0 & z & Z & Z & 屯 & ㄴ & Z & & 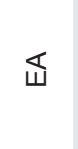 & ㄴ. & zu & & z & 凹 \\
\hline $\begin{array}{l}\frac{\pi}{0} \\
\frac{0}{2} \\
\frac{0}{0} \\
\frac{\pi}{0} \\
\frac{5}{0} \\
4\end{array}$ & $\begin{array}{l}\frac{0}{\sqrt{0}} \\
\Phi \\
\frac{0}{\omega} \\
\qquad\end{array}$ & $\begin{array}{l}\frac{0}{\pi} \\
\otimes \\
\otimes \\
\frac{0}{\omega}\end{array}$ & $\begin{array}{l}\frac{0}{\sqrt{0}} \\
\otimes \\
\frac{0}{0} \\
\qquad\end{array}$ & $\begin{array}{l}\text { O } \\
\text { ¿े } \\
0 \\
\otimes \\
\stackrel{0}{\Theta}\end{array}$ & $\begin{array}{l}0 \\
\stackrel{0}{0} \\
0 \\
0 \\
\stackrel{0}{0} \\
\stackrel{0}{0}\end{array}$ & 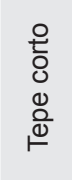 & $\begin{array}{l}\frac{0}{\pi} \\
\Phi \\
\frac{0}{0} \\
\stackrel{0}{0}\end{array}$ & $\begin{array}{l}\frac{0}{\pi} \\
\mathbb{0} \\
\frac{0}{0} \\
\qquad\end{array}$ & 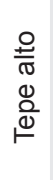 & 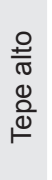 & $\begin{array}{l}\frac{0}{\pi} \\
\Phi \\
\frac{0}{0} \\
\stackrel{0}{0}\end{array}$ & 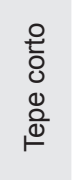 & 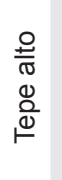 & & $\begin{array}{l}\frac{0}{0} \\
\frac{0}{0} \\
\frac{0}{0} \\
\frac{0}{0} \\
0\end{array}$ & $\begin{array}{l}\frac{0}{0} \\
\frac{0}{0} \\
\frac{0}{0} \\
0 \\
0\end{array}$ & $\begin{array}{l}\frac{0}{0} \\
\frac{0}{0} \\
\frac{0}{0} \\
\frac{0}{0} \\
0\end{array}$ & & 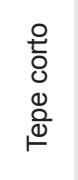 & 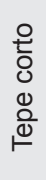 \\
\hline 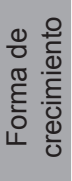 & $\begin{array}{l}\frac{0}{2} \\
\frac{0}{2} \\
\frac{\pi}{0} \\
\frac{0}{0} \\
\frac{0}{4}\end{array}$ & $\begin{array}{l}\frac{0}{0} \\
\frac{0}{20} \\
\frac{0}{0} \\
\frac{0}{0} \\
\frac{0}{4}\end{array}$ & $\begin{array}{l}\frac{0}{0} \\
\frac{0}{20} \\
\frac{\mathbb{0}}{0} \\
\frac{0}{0} \\
\frac{0}{<}\end{array}$ & 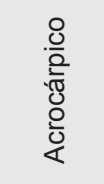 & 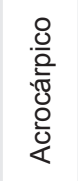 & $\begin{array}{l}\frac{0}{0} \\
\frac{0}{0} \\
.00 \\
\frac{0}{0} \\
\frac{0}{0}\end{array}$ & $\begin{array}{l}\frac{0}{0} \\
\frac{0}{0} \\
\frac{10}{0} \\
\frac{0}{0} \\
\frac{0}{4}\end{array}$ & $\begin{array}{l}\frac{0}{2} \\
\frac{0}{\pi} \\
\frac{\pi}{0} \\
\frac{0}{0} \\
\frac{1}{4}\end{array}$ & $\begin{array}{l}\frac{0}{2} \\
\frac{0}{2} \\
\frac{0}{0} \\
\frac{0}{0} \\
\frac{0}{4}\end{array}$ & $\begin{array}{l}\frac{8}{2} \\
\frac{0}{20} \\
\frac{0}{0} \\
\frac{0}{4}\end{array}$ & $\begin{array}{l}\frac{0}{0} \\
\frac{0}{2} \\
.00 \\
\frac{0}{0} \\
\frac{0}{4}\end{array}$ & $\begin{array}{l}\frac{0}{0} \\
\frac{0}{0} \\
.00 \\
\frac{0}{0} \\
\frac{0}{0}\end{array}$ & $\begin{array}{l}\frac{0}{0} \\
\frac{0}{20} \\
\frac{\pi}{0} \\
\frac{0}{0} \\
\frac{0}{4}\end{array}$ & & $\begin{array}{l}\frac{0}{0} \\
\frac{0}{2} \\
\frac{d}{0} \\
\frac{0}{3} \\
\frac{0}{0}\end{array}$ & $\begin{array}{l}\frac{0}{0} \\
\frac{0}{20} \\
\frac{0}{0} \\
\frac{0}{3} \\
\frac{\omega}{\alpha}\end{array}$ & $\begin{array}{l}\frac{0}{0} \\
\frac{0}{2} \\
\frac{\pi}{0} \\
\frac{0}{3} \\
\frac{\omega}{0}\end{array}$ & & $\begin{array}{l}\frac{0}{0} \\
\frac{0}{2} \\
\frac{\sqrt{0}}{0} \\
\frac{0}{3} \\
\frac{0}{0}\end{array}$ & $\begin{array}{l}\frac{8}{0} \\
\frac{0}{2} \\
\frac{0}{0} \\
\frac{0}{0} \\
\frac{0}{4}\end{array}$ \\
\hline 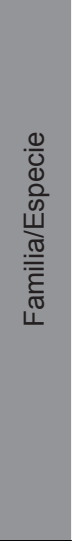 & 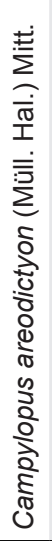 & 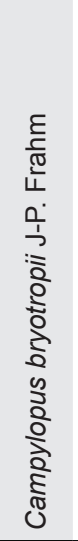 & 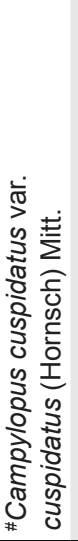 & 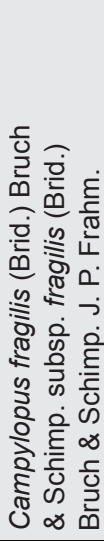 & 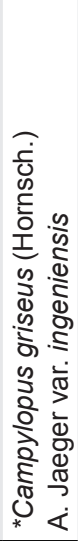 & 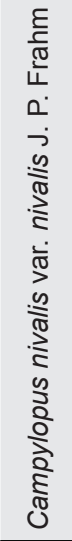 & 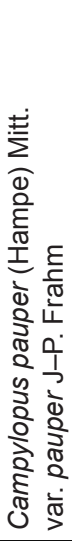 & 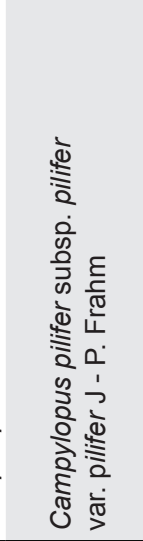 & 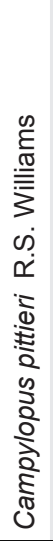 & 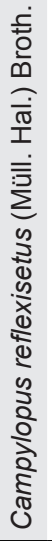 & 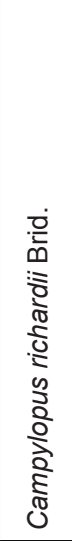 & 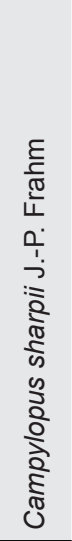 & 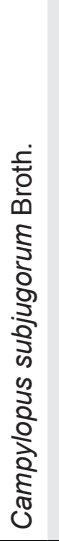 & 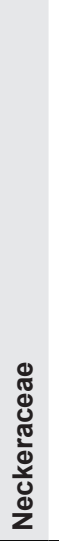 & 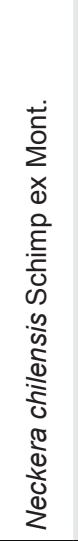 & 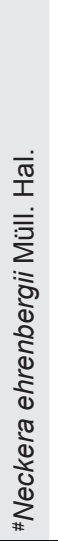 & 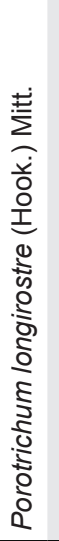 & 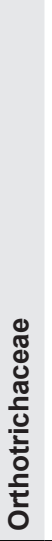 & 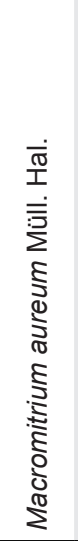 & 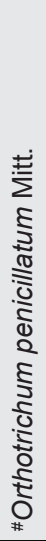 \\
\hline
\end{tabular}




\begin{tabular}{|c|c|c|c|c|c|c|c|c|c|c|c|c|c|c|c|c|c|c|c|c|c|c|}
\hline 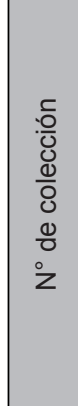 & 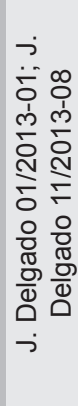 & 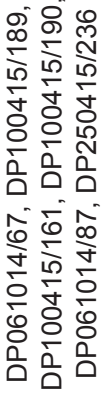 & 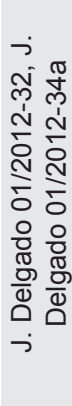 & 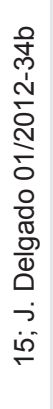 & & 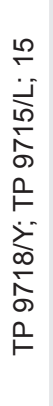 & & $\begin{array}{l}\text { D } \\
\stackrel{n}{f} \\
\dot{\gamma} \\
\dot{\gamma}\end{array}$ & & 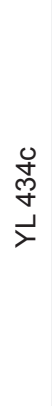 & 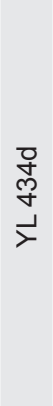 & 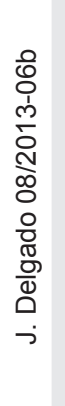 & 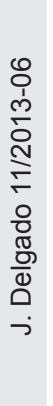 & 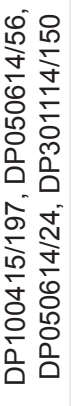 & $\stackrel{\stackrel{\rho}{\text { f }}}{\stackrel{\nu}{\succ}}$ & & 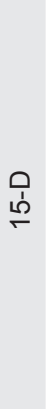 & 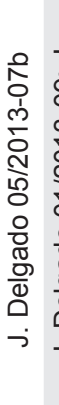 & 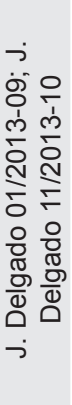 & 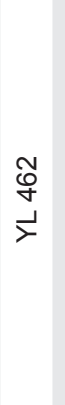 & 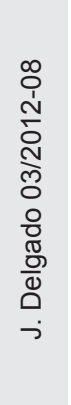 & 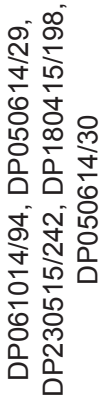 \\
\hline $\begin{array}{l}\stackrel{0}{\vec{D}} \\
\text { D } \\
\stackrel{0}{\infty}\end{array}$ & $\stackrel{\sim}{\sim}$ & م & $\stackrel{\stackrel{े}{m}}{\stackrel{5}{\sigma}}$ & $\sigma$ & & $\stackrel{N}{\sim}$ & & $m$ & & $r$ & - & $\stackrel{\nabla}{\oplus}$ & $\stackrel{\vec{m}}{\stackrel{m}{\sigma}}$ & م & $\stackrel{N}{\stackrel{2}{~}}$ & & $\stackrel{m}{\sim}$ & 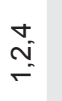 & $\stackrel{\stackrel{v}{N}}{\stackrel{n}{\leftarrow}}$ & $\nabla$ & $\begin{array}{l}\stackrel{+}{m} \\
\stackrel{\sim}{\sim} \\
\stackrel{2}{n}\end{array}$ & م \\
\hline 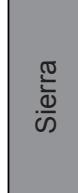 & $\frac{\frac{\pi}{\pi}}{\frac{\pi}{5}}$ & $\begin{array}{l}\frac{\pi}{0} \\
\frac{\pi}{\pi} \\
\frac{0}{2}\end{array}$ & $\frac{\frac{\pi}{\pi}}{\frac{\pi}{5}}$ & $\begin{array}{l}\frac{\pi}{0} \\
\frac{\pi}{J} \\
0 \\
\frac{\pi}{\pi} \\
\frac{\pi}{\pi} \\
\frac{0}{2} \\
\frac{2}{z}\end{array}$ & & $\begin{array}{l}\frac{\pi}{0} \\
\frac{\pi}{2} \\
\frac{1}{2}\end{array}$ & & $\begin{array}{l}\frac{\pi}{0} \\
\frac{\pi}{0} \\
\stackrel{0}{2}\end{array}$ & & $\begin{array}{l}\frac{\pi}{0} \\
\frac{\pi}{\pi} \\
\stackrel{0}{2}\end{array}$ & $\begin{array}{l}\frac{\pi}{0} \\
\frac{\pi}{2} \\
0 \\
2\end{array}$ & $\frac{\frac{\pi}{\pi}}{\frac{\pi}{5}}$ & $\frac{\frac{\pi}{\pi}}{\frac{\pi}{J}}$ & $\begin{array}{l}\frac{\pi}{0} \\
\frac{\pi}{2} \\
\mathbb{2} \\
z\end{array}$ & $\begin{array}{l}\frac{\pi}{0} \\
\frac{\pi}{0} \\
\frac{0}{2}\end{array}$ & & $\begin{array}{l}\frac{\pi}{0} \\
\frac{\pi}{\pi} \\
\frac{0}{2} \\
z\end{array}$ & $\frac{\frac{\pi}{\pi}}{\frac{\pi}{J}}$ & $\frac{\frac{\pi}{\pi}}{\frac{\pi}{5}}$ & $\begin{array}{l}\frac{\pi}{\pi} \\
\frac{\pi}{\pi} \\
0 \\
\frac{\pi}{\pi} \\
\frac{\pi}{\pi} \\
\frac{0}{2} \\
z\end{array}$ & $\frac{\frac{\pi}{\pi}}{\frac{\pi}{J}}$ & $\begin{array}{l}\frac{\pi}{0} \\
\frac{\pi}{\pi} \\
\frac{\pi}{2}\end{array}$ \\
\hline 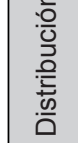 & 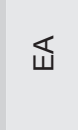 & ㄴ. & $\sum_{0}$ & 岀 & & z & & 0 & & zu & z & 岀 & 凹 & 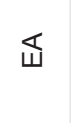 & $\sum_{\frac{0}{<}}$ & & Z & 0 & 0 & 0 & 그 & 岀 \\
\hline $\begin{array}{l}\frac{\pi}{0} \\
\frac{0}{2} \\
\frac{0}{0} \\
\frac{\pi}{0} \\
\sum_{0}^{0} \\
4\end{array}$ & 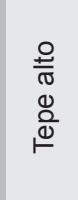 & 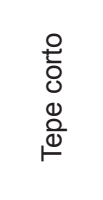 & 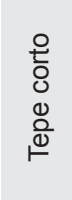 & 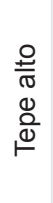 & & 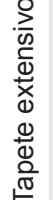 & & 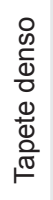 & & 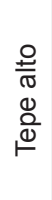 & $\begin{array}{l}\frac{0}{\pi} \\
\Phi \\
\frac{0}{0} \\
\qquad\end{array}$ & 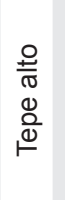 & $\begin{array}{l}\frac{0}{0} \\
\mathbb{0} \\
\frac{0}{0} \\
\stackrel{0}{0}\end{array}$ & $\begin{array}{l}\frac{0}{\pi} \\
\mathbb{0} \\
\mathbb{0} \\
\stackrel{0}{6}\end{array}$ & $\begin{array}{l}\frac{0}{\pi} \\
\mathbb{0} \\
\frac{0}{0} \\
\stackrel{0}{0}\end{array}$ & & 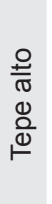 & $\begin{array}{l}\frac{0}{\sigma} \\
\Phi \\
\stackrel{0}{0} \\
\stackrel{\varrho}{\varrho}\end{array}$ & 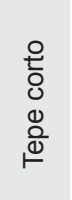 & 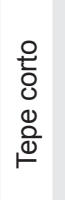 & $\begin{array}{l}\frac{0}{\pi} \\
\frac{0}{0} \\
\stackrel{0}{\oplus}\end{array}$ & $\begin{array}{l}\frac{0}{\pi} \\
\Phi \\
\frac{0}{\omega}\end{array}$ \\
\hline 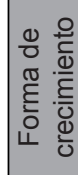 & $\begin{array}{l}0 \\
\frac{0}{0} \\
\frac{\pi}{0} \\
\frac{0}{0} \\
\frac{0}{0}\end{array}$ & 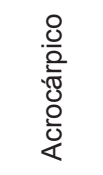 & $\begin{array}{l}\frac{0}{0} \\
\frac{0}{2} \\
\frac{\pi}{0} \\
\frac{0}{0} \\
\frac{0}{4}\end{array}$ & $\begin{array}{l}\frac{0}{0} \\
\frac{0}{0} \\
\text {.त्र } \\
\frac{0}{0} \\
\frac{0}{4}\end{array}$ & & 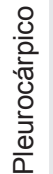 & & 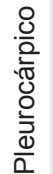 & & $\begin{array}{l}\frac{0}{0} \\
\frac{0}{2} \\
\text {. } \\
\frac{0}{0} \\
\frac{0}{0}\end{array}$ & 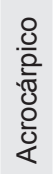 & 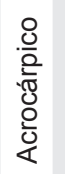 & $\begin{array}{l}\frac{.0}{0} \\
\frac{.0}{\pi} \\
\frac{0}{0} \\
\frac{0}{0}\end{array}$ & 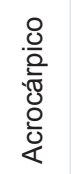 & 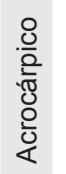 & & $\begin{array}{l}\frac{8}{0} \\
\text { 윰 } \\
\frac{0}{0} \\
\frac{0}{0}\end{array}$ & 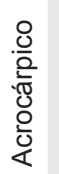 & $\begin{array}{l}\frac{0}{0} \\
\frac{0}{20} \\
\frac{\pi}{0} \\
\frac{0}{0} \\
\frac{0}{4}\end{array}$ & $\begin{array}{l}\frac{0}{0} \\
\frac{0}{2} \\
\frac{\pi}{0} \\
\frac{0}{0} \\
\frac{0}{4}\end{array}$ & $\begin{array}{l}\text { 을 } \\
\frac{0}{0} \\
. \overline{0} \\
\frac{0}{0} \\
\frac{0}{4}\end{array}$ & $\begin{array}{l}\frac{0}{0} \\
\frac{0}{2} \\
\frac{.0}{0} \\
\frac{0}{0} \\
\frac{0}{4}\end{array}$ \\
\hline 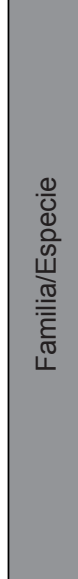 & 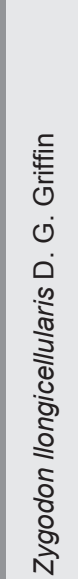 & 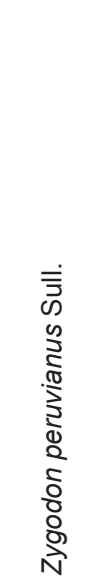 & 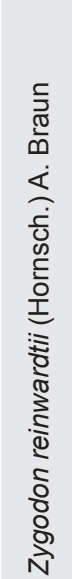 & 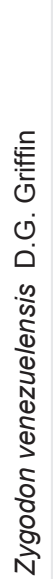 & 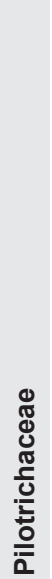 & 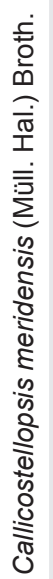 & 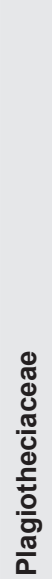 & 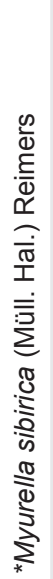 & 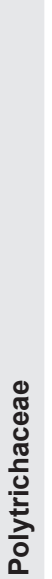 & 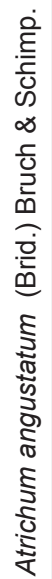 & 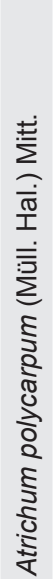 & 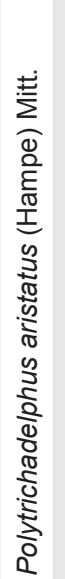 & 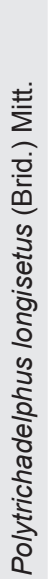 & 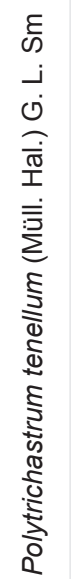 & 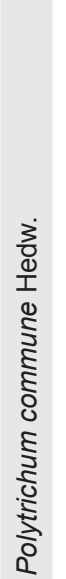 & 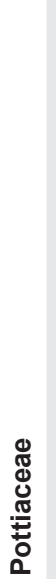 & 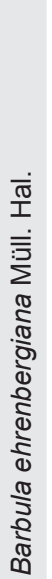 & 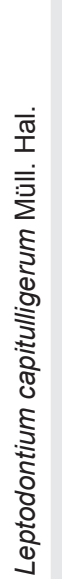 & 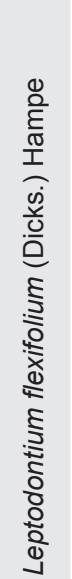 & 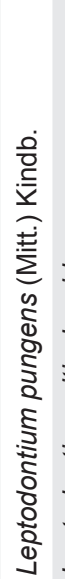 & 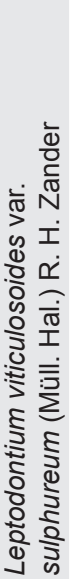 & 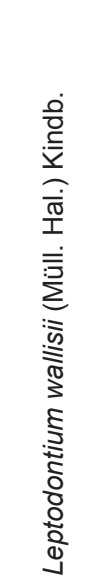 \\
\hline
\end{tabular}




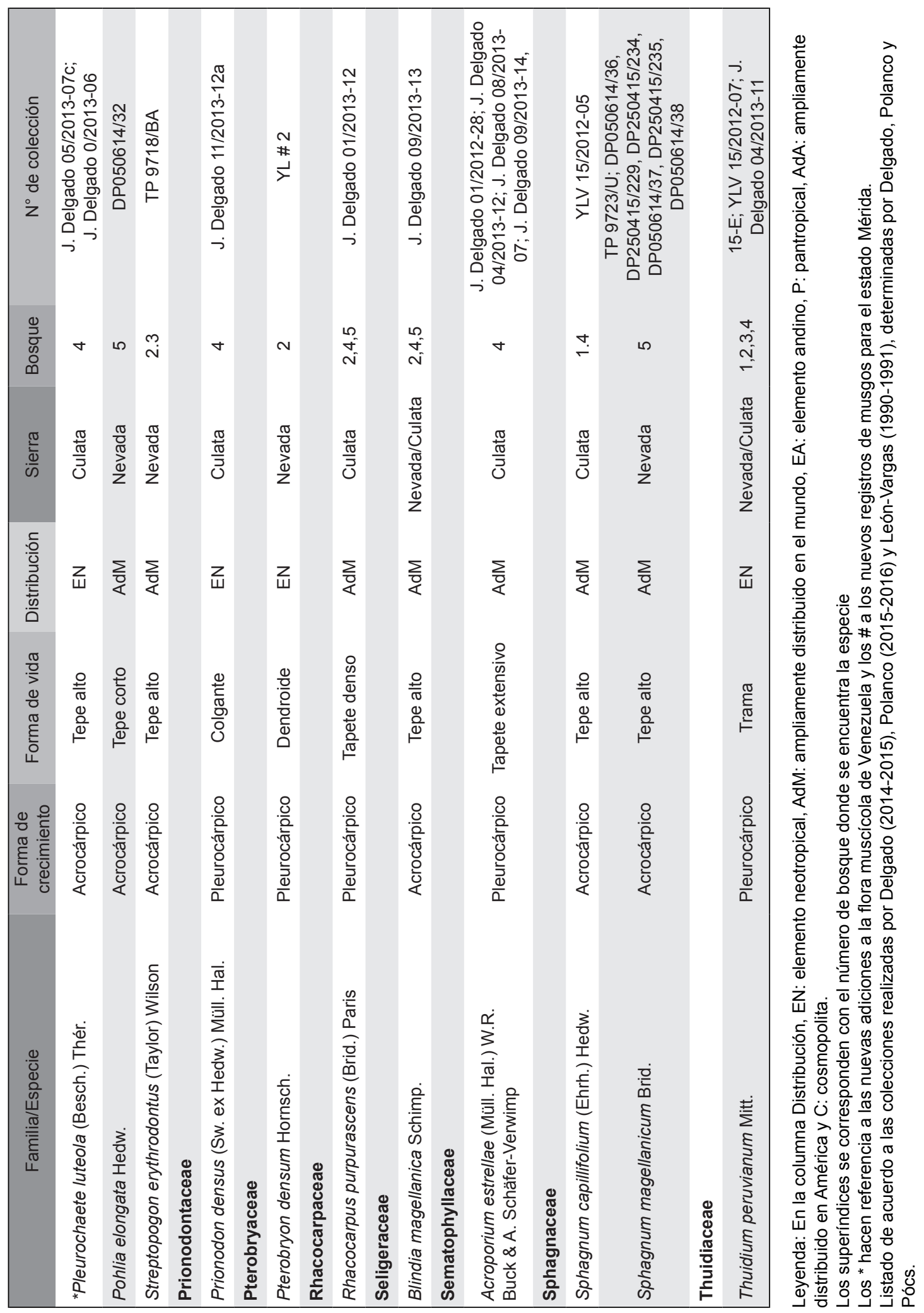


Bol. Soc. Argent. Bot. 52 (2) 2017

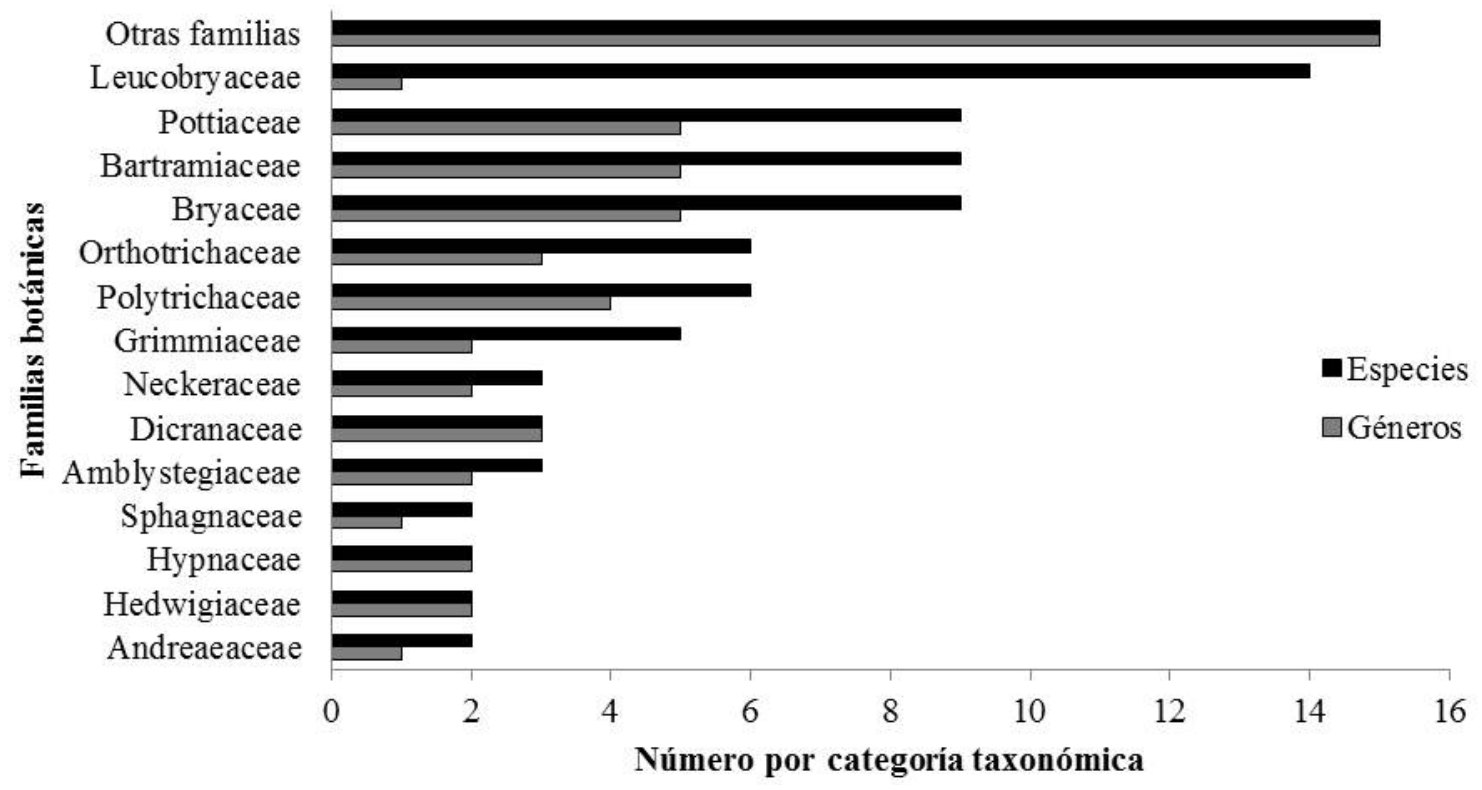

Fig. 2. Riqueza específica y genérica de las familias de musgos de los parches de bosques de Polylepis del estado Mérida, Venezuela.

El bosque más diverso es el de la Culata (parche 4), con 52 especies distribuidas en 36 géneros y 22 familias. Le sigue el bosque 2, con 45 especies (31 géneros/21 familias) y finalmente los parches $1,3 \mathrm{y}$ 5 con 43, 29 y 23 especies respectivamente (Tabla 3), todos estos ubicados en la Sierra Nevada de Mérida. En la Figura 3, se muestra la diversidad de especies de las 4 familias comunes y más importantes en cada uno de los parches de bosque, siendo la familia Leucobryaceae la más diversa en todos a excepción del parche 2, donde Bartramiaceae es la familia dominante.

De manera general, los géneros más diversos a nivel específico son Campylopus (14 especies), Leptodontium (5), Breutelia (4), Bryum (4), Zygodon (4) y Racomitrium (3 especies). Los géneros restantes

\begin{tabular}{|c|c|c|c|c|c|}
\hline & Bosque & Bosqu & Bosque & Bosque & Bosque \\
\hline & 1 & 2 & 3 & 4 & 5 \\
\hline Familias & 19 & 21 & 16 & 22 & 14 \\
\hline Géneros & 28 & 31 & 22 & 36 & 19 \\
\hline Especies & 43 & 45 & 29 & 52 & 23 \\
\hline
\end{tabular}

están constituidos por 2 y 1 especie. Por parche de bosque, la tendencia a la predominancia del género Campylopus se mantiene, donde la riqueza específica de éste oscila entre 4 y 8 especies en los bosques 5 y 1 respectivamente. Sin embargo, se evidencian diferencias en términos de composición florística entre ellos, a excepción de los bosques 1 y 2 , donde predominan los mismos géneros. En los bosques 3, 4 y 5, el género Breutelia es desplazado y el recambio por géneros como Racomitrium (bosques 3 y 5) y Zygodon (bosque 4) se evidencia (Figura 4).

Formas de vida y formas de crecimiento

Los tepes son las formas de vida dominantes, siendo los tepes altos los más abundantes con un $41,11 \%$ (37 especies). Por su parte, los tepes cortos representan un $24,44 \%$ del total (22 especies), seguido por especies formadoras de tapetes extensivos con un $8,89 \%$ (8 especies). Las tramas y las formas colgantes no son tan frecuentes en los bosques de Polylepis estudiados. Del total, 68 especies (75,6 \%) son acrocárpicas, mientras que las 22 especies $(24,4$ $\%$ ) restantes son pleurocárpicas (Figura 5).

\section{Análisis fitogeográfico}

Desde el punto de vista fitogeográfico, los bosques de Polylepis albergan una gran variedad de musgos distribuidos en 6 regiones (Figura 6). Los elementos 


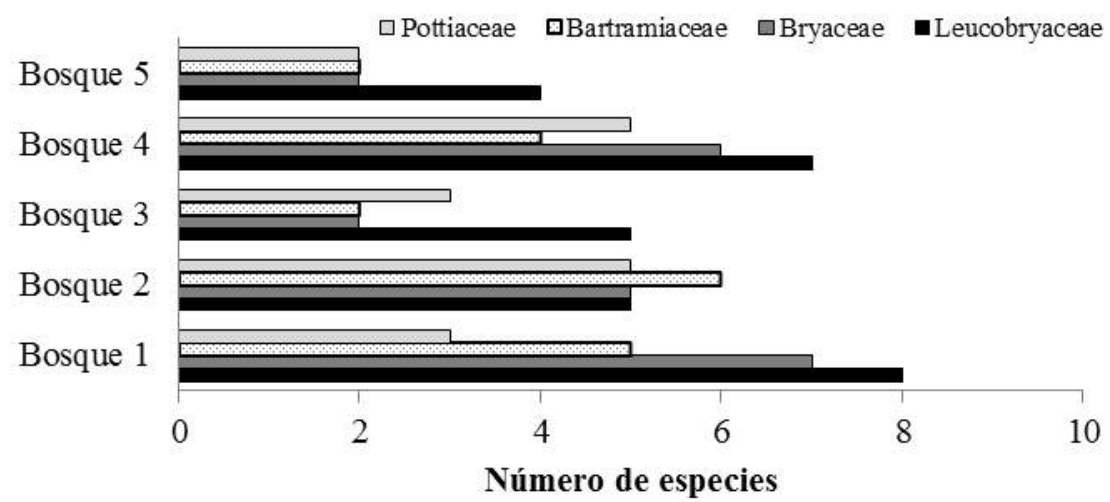

Fig. 3. Número de especies de las familias comunes y más diversas en los parches de bosques de Polylepis.

neotropicales (EN) son los más comunes con el $36 \%$ de las especies (32 especies), seguido por los elementos ampliamente distribuidos en el mundo (AdM) con un $27 \%$, y por ultimo las especies andinas (EA) con un $18 \%$. De estas últimas, Campylopus griseus (Hornsch.) A. Jaeger var. ingeniensis figura como un nuevo registro para la flora muscícola de Venezuela (Polanco \& León, en revisión), Orthotrichum penicillatum Mitt. como especie de distribución restringida en páramos de Colombia y Venezuela y Zygodon longicellularis D. G. Griffin y Z. venezuelensis D.G. Griffin, como endémicas de los páramos venezolanos (Griffin, 1990; Churchill et al. 2000).

\section{Afinidades floristicas}

Al comparar la brioflora de los parches de bosque de Polylepis inventariados (Figura 7), se

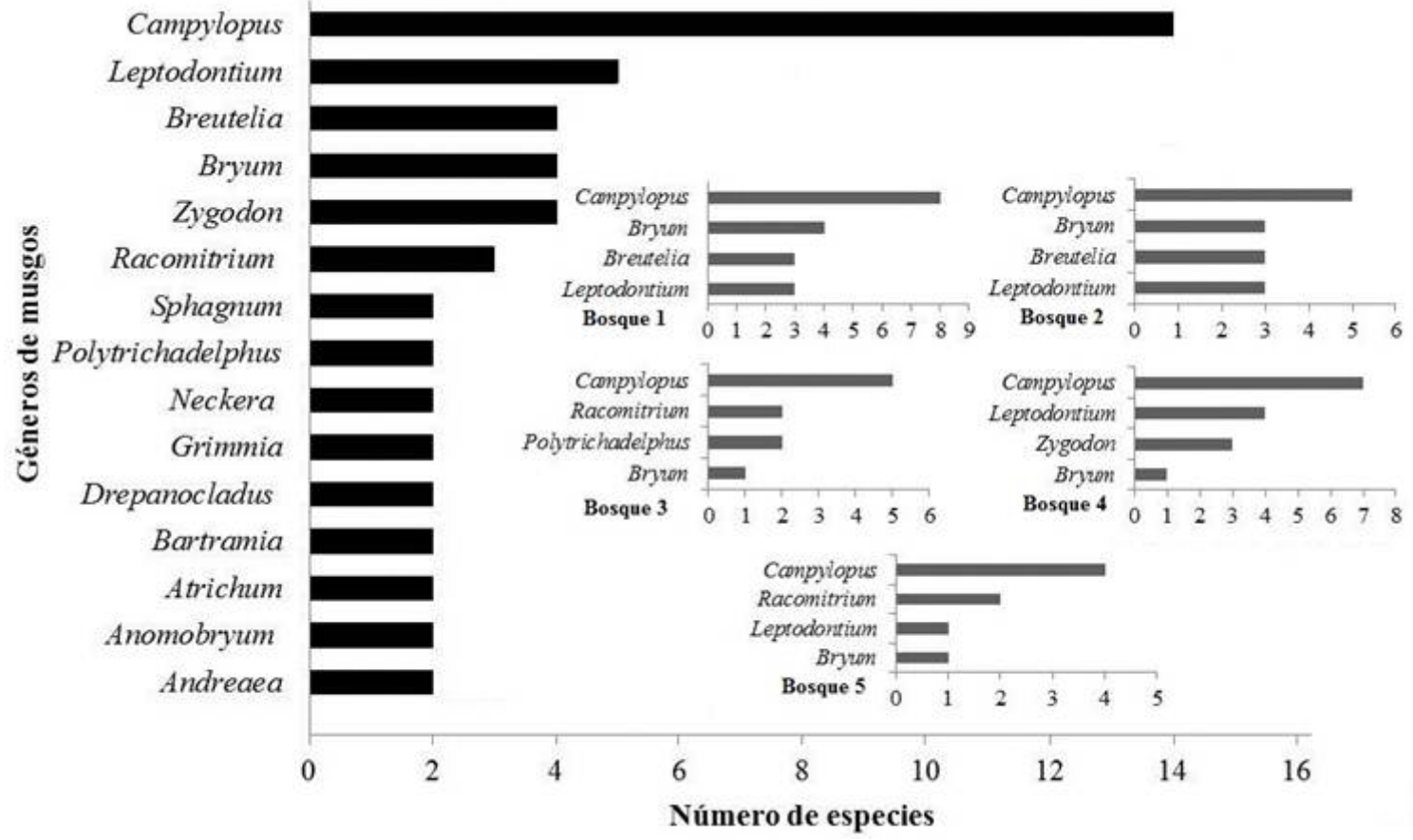

Fig. 4. Géneros de musgos más diversos en los parches de bosques de Polylepis. 
Bol. Soc. Argent. Bot. 52 (2) 2017

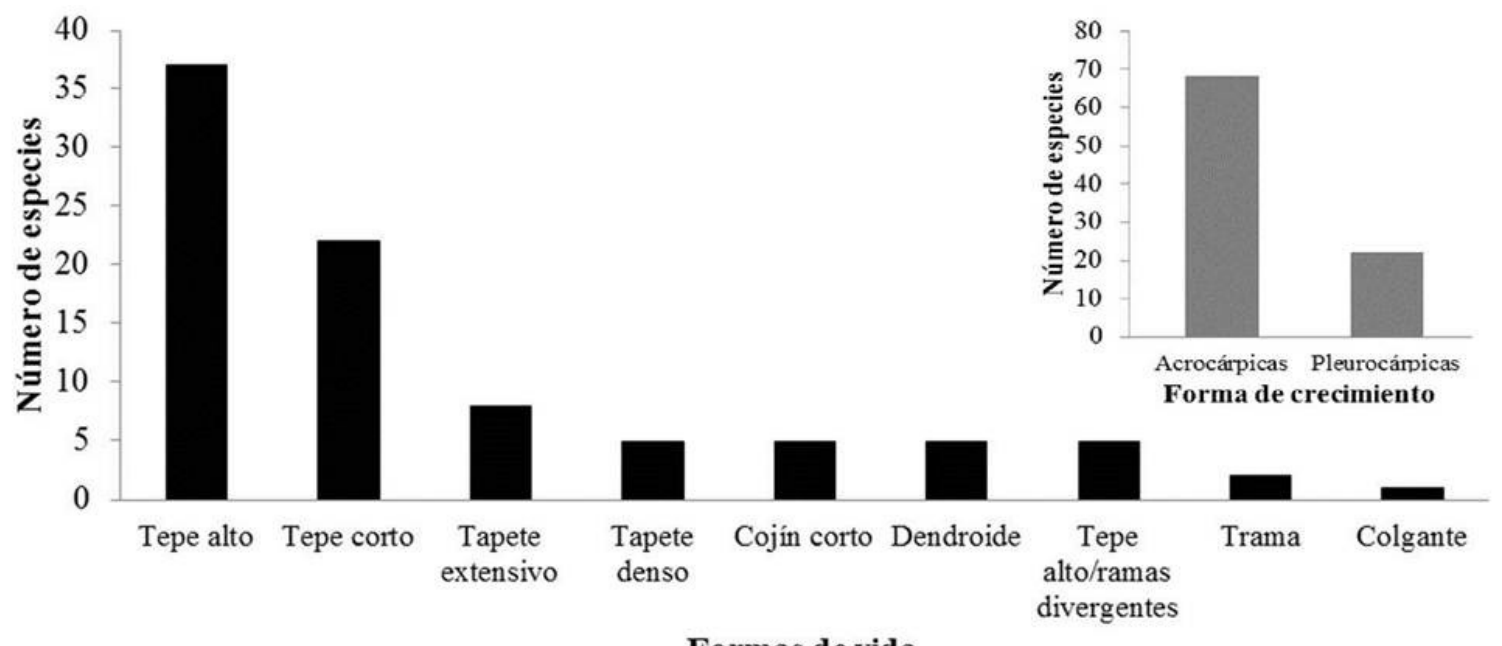

Formas de vida

Fig. 5. Formas de vida y de crecimiento de los musgos encontrados en los parches de bosques de Polylepis inventariados.

observa que los parches más afines entre sí, son los 1 y 2 (Sierra Nevada) que comparten el 61,4\% de sus especies. El parche 4 (Culata) comparte el $56,8 \%$ de las especies con el parche 1 , y el $57,7 \%$ con el parche 2 . Los parches de bosque más disímiles con el resto son los parches 3 y 5 (Sierra Nevada), donde el primero comparte el $50 \%$ de las especies con el grupo formado por los parches 1,2 y 4; mientras que el segundo (parche 5), comparte menos del $30 \%$ de sus especies con el resto.

Nuevos reportes

AMBLYSTEGIACEAE

Drepanocladus sordidus (Müll. Hal.) Hedenäs

Musgo pleurocárpico, formando tapetes extensivos. Hojas falcado-secundas con costa

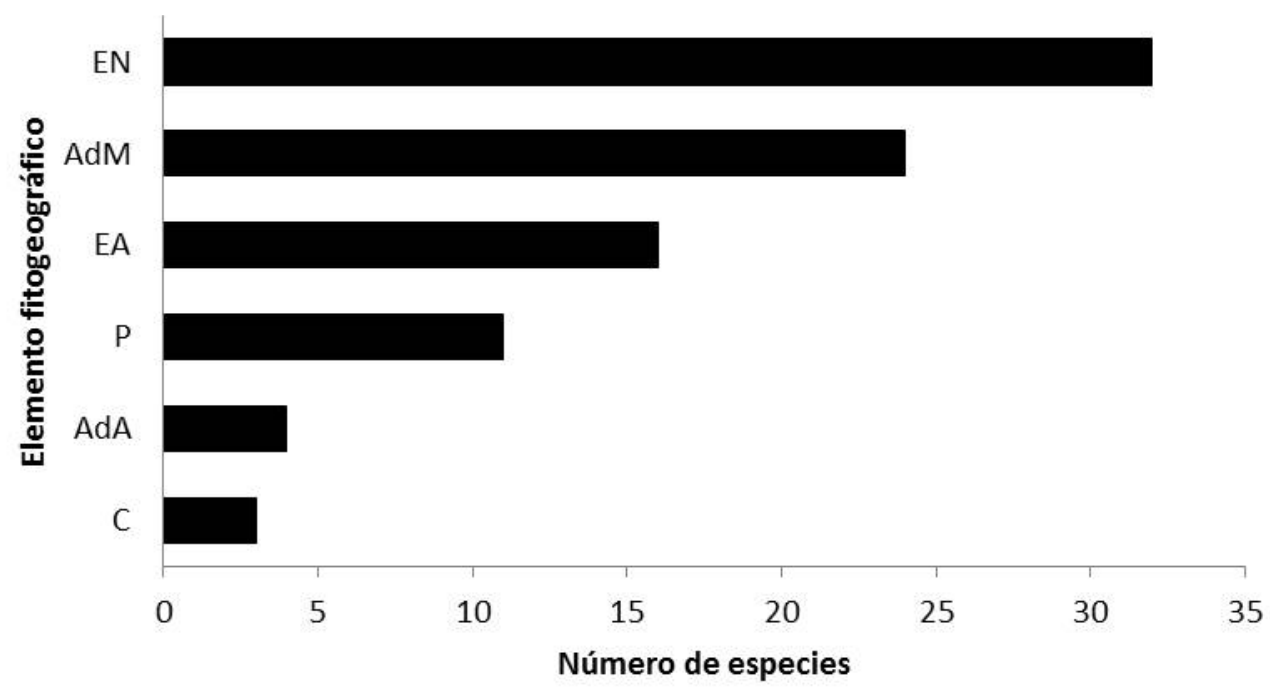

Fig. 6. División fitogeográfica de las especies de musgos encontradas en los parches de bosques de Polylepis (EN: neotropical; AdM: ampliamente distribuido en el mundo; EA: elemento andino; P: pantropical; AdA: ampliamente distribuido en América y C: cosmopolita). 


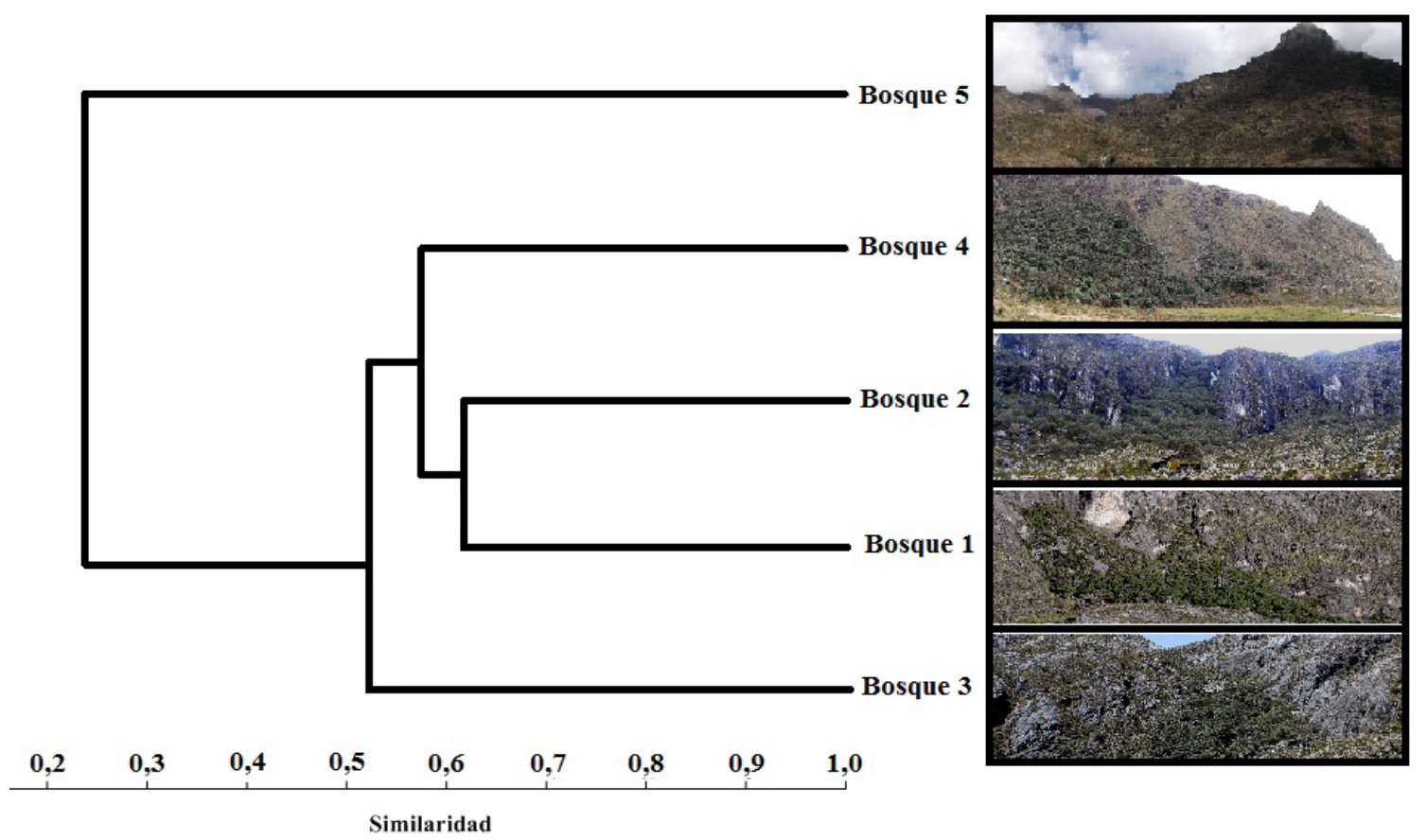

Fig. 7. Cluster de similaridad florística entre los parches de bosques de Polylepis inventariados en el estado Mérida.

terminando muy por debajo del acumen, células alares extendiéndose hasta la mitad de la distancia entre la costa y el margen. Bosque 2, Estación Loma Redonda, Sistema Teleférico Mukumbarí, $8^{\circ} 32^{\prime} \mathrm{N}, 71^{\circ} 04,5^{\prime} \mathrm{W}, 3850$ - $4070 \mathrm{~m}$, León, Y. Material estudiado: YL434e (MERC) (Det. Delgado, J. 2016). Especie típica del páramo y la puna húmeda, creciendo a orillas de cursos de aguas, en lugares cenagosos. Reportada para Colombia (3400-3950 m), Ecuador (2500-3950 $\mathrm{m})$, Perú (3650-4350 m) y Bolivia (3800-4680 m). Distribución: Antillas (Jamaica), México, Andes, Norteamérica y Europa (Churchill et al. 2000).

\section{PLAGIOTHECIACEAE}

Myurella sibirica (Müll. Hal.) Reimers

Musgo pleurocárpico, formador de densos tapetes, verde-glaucos. Hojas ampliamente espaciadas, con márgenes irregularmente dentados a espinosamente dentados; células unipapilosas. Bosque 3, Estación Loma Redonda, Sistema teleférico de Mérida, Sierra Nevada, 8³2'11,33” N, 7104'44,06" W, 4122 m, Y. León. Material estudiado: YL475d (MERC) (Det. J. Delgado,
2016). Especie común en rocas calcáreas y suelos húmedos calcáreos, sobre todo en las zonas boreales. Distribución: Norteamérica, Europa y Asia (Crum \& Anderson, 1981).

\section{POTTIACEAE}

Pleurochaete luteola (Besch.) Thér.

Musgo acrocárpico formador de tepes altos. Hojas apretadas desde una base anchamente amplectante, crispado a contortas en seco, patentes a escuarroso - recurvadas en húmedo; lámina con células de la margen formando una "V". Bosque 4, Páramo El Escorial, Sierra de La Culata, Laguna "La Barrosa", 844’34,30" N, 7102'45,31"W, 3618 m, 10/03/2013 y 17/04/2013, J. Delgado. Material estudiado: J. Delgado 05/2013-07c (MERC), J. Delgado 0/2013-06 (MERC) (Det. Delgado, J. 2016). Especie típica de vegetación seca, montana abierta o de bosques espinosos en sitios parcialmente sombreados o expuestos, sobre suelos o rocas. Distribución: se conoce en Colombia (1800-2500 m), Ecuador (1800-1970 $\mathrm{m})$, Perú (2800-4000 m) y Bolivia (1420-2050 m) y noroeste de Argentina (2060 m) (Churchill et al. 2000). 
Además de estas especies, Polanco \& León (en revisión) reportan para el bosque 5 ubicado en el Valle morrénico de Mucubají, las especies Drepanocladus polygamus (Schimp.) Hedenäs y Campylopus griseus var. ingeniensis (R.S. Williams) J.-P. Frahm como nuevas adiciones a la brioflora en Venezuela.

Así mismo, las especies Leiomela bartramioides (Hook.) Paris, Fabronia ciliaris var. polycarpa (Hook.) W.R. Buck, Campylopus cuspidatus var. cuspidatus (Hornsch) Mitt., Neckera ehrenbergii Müll. Hal, Acroporium estrellae (Müll. Hal.) W.R. Buck \& Schäf.-Verw. y Orthotrichum penicillatum Mitt., son adiciones a la flora de musgos del Estado Mérida.

\section{Discusión}

Para los parches de bosque de Polylepis en Venezuela se tiene el reporte de 90 especies de musgos, distribuidas en 53 géneros y 29 familias. De estas, las especies Drepanocladus sordidus (Müll. Hal.) Hedenäs, Myurella sibirica (Müll. Hal.) Reimers y Pleurochaete luteola (Besch.) Thér, se adicionan a la flora muscícola de Venezuela, así como nueve nuevos registros para el estado Mérida.

De manera general, las familias Leucobryaceae (14 especies), Pottiaceae (9), Bartramiaceae (9), Orthotrichaceae (9), Bryaceae (6) y Polytrichaceae (6), son las más diversas a nivel específico, distribuyéndose estas desde los bosques andinos de mediana altitud hasta los diferentes pisos parameros (Churchill \& Linares, 1995). Para Frahm (1991), Zander (1993) y Gradstein et al. (2001), estas son las familias más diversas en los Andes tropicales, cuyos miembros presentan características morfoanatómicas que les permiten colonizar una amplia gama de microhábitats; entre estas características figuran la presencia de mamilas o papilas en una o ambas superficies foliares, células basales alares hialinas, células guía y estereidas en la costa y una banda central en los tallos. La dominancia de los géneros Campylopus (14 especies), Leptodontium (5), Breutelia (4), Bryum (4) y Zygodon (4), son una muestra del éxito de estas familias de musgos en estos ambientes.

Para la Sierra Nevada de Mérida, 39 especies son exclusivas y para la Sierra de La Culata, 34. Del total, solo el $19 \%$ de las especies es compartido por ambas Sierras. Esta exclusividad de taxa sugiere un recambio en la composición de especies entre estos, la cual podría estar influenciada por la alta variabilidad ambiental a escala regional, local y temporal en el páramo andino (Monasterio \& Reyes, 1980). Las diferencias en términos de la composición florística en cada parche puede estar asociada a diferencias en la altitud, exposición, incidencia de radiación solar, humedad relativa, cobertura de dosel, pendiente y otros factores físicos que podrían estar determinando la colonización de estos ambientes por las diferentes especies de musgos.

Del total de musgos, algunas especies son frecuentes en los bosques de Polylepis. Por ejemplo, las especies Lepyrodon tomentosus (Hook.) Mitt. y Leptodontium wallisii (Müll. Hal.) Kindb., son comunes tanto en bosques de Polylepis venezolanos como en aquellos inventariados por Wolf (1993) en Colombia, Parolly \& Kürschner (2005) en el Ecuador y Aldana (2008) en Bolivia. Otras especies comunes en todos estos bosques son Bryum argenteum Hedw., Campylopus areodictyon (Müll. Hal.) Mitt., C. pilifer subsp. pilifer var. pilifer J - P. Frahm, C. pittieri R.S. Williams, Hedwigidium integrifolium (P.-Beauv.) Dixon in C. E. O. Jensen, Leptodontium capitulligerum Müll. Hal., L. viticulosoides var. sulphureum (Müll. Hal.) R. H. Zander, Rhodobryum grandifolium (Taylor) Schimp., Thuidium peruvianum Mitt. y Zygodon reinwardtii (Hornsch.) A. Braun (Wolf, 1993; Parolly \& Kürschner 2005; Aldana, 2008).

En los bosques de Polylepis inventariados en Venezuela (León, 1991; Delgado, 2015) los elementos neotropicales son los más comunes (32 especies, $36 \%$ del total). Delgado (2015), en su estudio de la flora vascular asociada a estos enclaves forestales, encuentra una predominancia de los elementos neotropicales, sobre los templados y cosmopolitas. Las condiciones en las cuales se establecen los bosques de Polylepis en la alta montaña, está muy asociada a la presencia de rocas, afloramientos o derrubios rocosos (Kleier \& Lambirinos, 2005), que determinan temperaturas ligeramente más cálidas en comparación con el páramo abierto, produciendo un efecto buffer (amortiguador) ante oscilaciones microclimáticas.

En conclusión, existe una alta diversidad de especies de musgos asociadas a bosques de Polylepis. Muchas de estas podrían ser especies exclusivas que podrían estar respondiendo a cambios, por lo que se hace necesario el monitoreo. En el marco 


\section{J. Delgado y Y. León - Musgos de los bosques de Polylepis en Venezuela}

de la conservación de los bosques de Polylepis en Suramérica y en el contexto del monitoreo de los efectos del cambio climático global, países como Colombia, Ecuador, Perú y Bolivia, han desarrollado estrategias y lineamientos de conservación de los bosques de Polylepis. En Venezuela, aunque los bosques de coloradito tienen una figura de alta relevancia ambiental, no cuentan con un sistema de protección monitoreo adecuados.

La implementación de planes de zonificación participativa de los remanentes de bosques deteriorados por la extensión de la línea de cultivo agrícola y zonas de pastoreo, la preservación de relictos de bosques mejorando el control de visitas a través de senderos de acceso en áreas protegidas ubicadas dentro de Parques Nacionales, la elaboración de listados de especies de plantas y animales en estos bosques y el desarrollo de programas de educación ambiental usando a los bosques de Polylepis como aula abierta, se sugieren como herramientas para promover la conservación de estos majestuosos oasis de vegetación en el páramo andino.

\section{Agradecimientos}

Agradecemos al CDCHTA de la Universidad de Los Andes (ULA) proyecto C-1884-14-01F y al Instituto Jardín Botánico de Mérida de la Facultad de Ciencias-ULA por el apoyo para la realización de estos inventarios y a los Ingenieros Annie Lobo de la Oficina de Gestión Ambiental del Sistema Teleférico Mukumbarí-Mérida y John Parra, quienes participaron en la logística de los últimos muestreos en campo en la Sierra Nevada de Mérida. Al Dr. Guillermo Suárez por su amable invitación a participar en este número especial en honor a nuestra querida Dra. María Magdalena Schiavone (Magui) quien ha realizado importantes aportes al conocimiento de la brioflora argentina y ha contribuido así al desarrollo de la briología en Latinoamérica.

\section{Bibliografía}

ALDANA, C. 2008. Briofitas de los bosques de Polylepis pepei (Provincia Murillo, La Paz - Bolivia): Comunidades y Ecología. Tesis de Licenciatura. Universidad Mayor de San Andrés, La Paz, Bolivia.
ARNAL, H. 1983. Estudio ecológico del bosque altiandino de Polylepis sericea Wedd. en la Cordillera de Mérida. Trabajo especial de grado. Universidad Central de Venezuela, Caracas.

CHURCHILL, S. P. \& E. LINARES. 1995. Prodomus Bryologiae Novo Granatensis: Introducción a la Flora de los musgos de Colombia. Biblioteca José Jerónimo Triana. Instituto de Ciencias Naturales, Universidad Nacional de Colombia. 12: 1-924.

CHURCHILL, S.P., GRIFFIN, D. III \& J. MUÑOZ. 2000.: A checklist of the mosses of the Tropical Andean countries. Monografías del Real Jardín Botánico. Ruizia 17: 1-203.

CRUM, H. A. \& ANDERSON, L. E. 1981. Mosses of eastern North America. The New York Botanical Garden. 69: 1-1328.

DELGADO, J. 2015. Flórula vascular y Bryophyta sensu stricto de un bosque de Polylepis sericea Wedd., ubicado en la sierra de la Culata, MéridaVenezuela. Trabajo especial de grado. Departamento de Biología. Universidad de Los Andes, Mérida, Venezuela.

FRAHM, J. P. 1991. Dicranaceae: Campylopodioideae, Paraleucobryoideae. Fl. Neotrop. Monogr. 54: 1-238.

FRAHM, J. P. 2003. Manual of Tropical Bryology. Trop. Bryol. 23: 9-195.

FRANSEN, S. 2005. Taxonomic revision of the moss genus Bartramia Hedw. Sections Bartramia and Vaginella C. Müll. Doctoral dissertation Göteborg University, Faculty of Science.

GARECA, E., HERMY, M., FJELDSA, J. \& O. HONNAY. 2010. Polylepis woodland remnants as biodiversity islands in the Bolivian high Andes. Biodivers. Conserv. 19: 3327-3346.

GOFFINET, B. \& J. SHAW. 2009. Classification: mosses [online]. University of Connecticut. Disponible en http://bryology.uconn.edu/classification-2/. [Acceso: Noviembre-Diciembre 2016]

GRADSTEIN, R., CHURCHILL, S., Y N. SALAZAR. 2001. Guide to the Bryophytes of Tropical America. Memoirs of the New York Botanical Garden 86: $1-577$.

GRIFFIN, D. 1981. El género Sphagnum en los Andes de Colombia y Venezuela. Clave para las especies frecuentes $\mathrm{u}$ ocasionales con notas ecológicas y taxonómicas. Cryptogamie, Bryol. Lichénol. 2:201211.

GRIFFIN, D. 1990. Two news pentastichous species of Zygodon from high elevation in Venezuela. Cryptogamie, Bryol. Lichénol. 11 (2):163-168.

HAMMER, ̌̌.; HARPER, D.A.T.; RYAN, P.D. 2001. PAST: Paleontological statistics software package for education and data analysis. Palaeontologia Electronica 4(1): 9pp. http://palaeo-electronica. org/2001_1/past/issue1_01.htm 
HEDENÄS, L. 2003. Amblystegiaceae (Musci). Flora Neotropica. The New York Botanical Garden 89: $1-108$.

HOCH, G. \& C. KÖRNER. 2005. Growth, demography and carbon relations of Polylepis trees at the world's highest treeline. Funct. Ecol. 19: 941-951.

HUECK, K. 1962. Gesellschaftsanschluss der Lärche und Grundlagen ihrer Natürlichen Verbreitung in den Ostalpen, Der Polylepsis - Wal in den Venezolanischen Anden, eine Parallele zum Mitteleurpäischen Latschenwald Angewandte. Pflanzensoziologie 17:57-76.

KESSLER, M. 2002. The "Polylepis problem": where do we stand? Ecotropica 8: 97-110.

KESSLER, M. 2006. Bosques de Polylepis. In: MORAES, M., ØLLGAARD, B., KVIST, L. P., BORCHSENIUS, F. \& H. Balslev (eds.). Botánica Económica de los Andes Centrales. Pp. 110-120. Universidad Mayor de San Andrés, La Paz.

KESSLER, M. \& A. N. SCHMIDT-LEBUHN. 2006. Taxonomical and distributional notes on Polylepis (Rosaceae). Org. Divers. \& Evol. 6, Electr. Suppl. $1: 1-10$

KLEIER C. \& J. LAMBIRINOS. 2005. The importance of nurse associations for three tropical alpine life forms. Arct. Antarct. Alp Res. 37 (3): 320-334.

KÖRNER, CH. \& PAULSEN, J. 2004. A world-wide study of high altitude treeline temperaturas. $J$. Biogeogr. 31: 713-732.

LEÓN, Y. 1991. Estudio de la vegetación vascular de tres bosques de Polylepis sericea Wedd. ubicados en la Sierra Nevada de Mérida. Trabajo especial de grado. Departamento de Biología. Universidad de Los Andes. Mérida - Venezuela.

LEÓN, Y., USSHER, M. S., ROJAS, C. \& J. DELGADO. [en línea]. Base de datos de los musgos de Venezuela (MdV). Disponible en http://musgos. cecalc.ula.ve/. [Acceso: enero 2014-diciembre 2016]

LEWINSKY, J. 1993. Orthotrichum spanotrichum Lewinsky, sp. nov. from Venezuela. Lindbergia 18:116-120.

MARCORA, P., HENSEN, I., RENISON, D. SELTMANN, P. \& K. WESCHE. 2008. The performance of Polylepis australis trees along their entire altitudinal range: implications of climate change for their conservation. Divers. Distrib. 14: 630-636.

MENDOZA, W. \& A., CANO. 2011. Diversidad del género Polylepis (Rosaceae, Sanguisorbeae) en los Andes peruanos. Rev. peru. biol. 18 (2): 197-200.

MENDOZA, W. 2010. Taxonomía y distribución de las especies peruanas de Polylepis Ruíz \& Pav. (Rosaceae, Magnoliopsida). Tesis de Magister. UNMSM. Lima-Perú. 125 p.
MONASTERIO, M. 1980a. Los Páramos Andinos como Región Natural. Características Biogeográficas Generales y Afinidades con otras Regiones Andinas. In: MONASTERIO, M. (ed.): Estudios Ecológicos en los Páramos Andinos. Pp. 15-27. Ediciones de la Universidad de Los Andes. Mérida, Venezuela.

MONASTERIO, M. 1980b. Las Formaciones Vegetales de los Páramos de Venezuela. In: MONASTERIO, M. (ed.): Estudios Ecológicos en los Páramos Andinos. Pp. 93-158. Ediciones de la Universidad de Los Andes. Mérida, Venezuela.

MONASTERIO, M. \& S. REYES. 1980. Diversidad Ambiental y Variación de la Vegetación en los Páramos de Los Andes Venezolanos. In: MONASTERIO, M. (ed.): Estudios Ecológicos de los Páramos Andinos. Pp. 47-91. Ediciones de la Universidad de Los Andes., Mérida, Venezuela.

MUÑOZ, J. 1999. A revision of Grimmia (Musci, Grimmiaceae) in the Americas. 1: Latin America. Ann. Missouri Bot. Gard. 86: 118-191.

NAVARRO, G., MOLINA, J. A. \& N. DE LA PARRA. 2005. Classification of the high - Andean Polylepis forest in Bolivia. Plant Ecol. 176: 113-130.

OCHI, H. 1980. A Revision of the Neotropical Bryoideae, Musci (First Part). J. Fac. Educ. Tottori Univ. Nat. Sci. 29: 49-154.

OCHI, H. 1981. A revision of the Neotropical Bryoideae, Musci (Second Part). J. Fac. Educ. Tottori Univ. Nat. Sci. 30: 21-55.

PAROLLY, G.\&H.KÜRSCHNER. 2005. Syntaxonomy, life forms, life strategies and ecomorphology of the subandean woodlands and Polylepis forest in Central Ecuador. Ecosociological studies in Ecuadorian bryophyte communities. 6. Bot. Jahrb. Syst. 126 (2): 211-252.

POLANCO, D. 2015. Musgos del complejo morrénico de Mucubají, Parque Nacional Sierra Nevada, Mérida, Venezuela. Trabajo Especial de Grado. Departamento de Biología. Universidad de Los Andes, Mérida, Venezuela.

POLANCO, D. \& Y. LEÓN. 2016. Nuevos registros de musgos del complejo morrénico de Mucubají, Parque Nacional Sierra Nevada, Mérida, Venezuela. Enviado a ґActa Bot. Venez.

RENISON, D. \& A. M. CINGOLANI. 1998. Experiencias en germinación y reproducción vegetativa aplicados a la reforestación con Polylepis australis Bitt. (Rosaceae) en las Sierras Grandes de Córdoba, Argentina. Agriscientia 15: 47-53.

SCHOFIELD, W. B. 1992. Bryophyte distribution patterns. In: BATES, J. W. \& A. M. FARMER (eds.): Bryophytes and Lichens in Changing World. Pp. 103-130. Oxford: Clarendon Press. 


\section{J. Delgado y Y. León - Musgos de los bosques de Polylepis en Venezuela}

SCHUBERT, C. \& S. VALASTRO. 1973. Páramo de La Culata, Estado Mérida: Glaciación del Pleistoceno Tardío. Asoc. Venez, de Geol. Min y Petrol. Boletín Informativo 16:108-142.

SHARP, A. J., CRUM, H. \& P. ECKEL. 1994. The Moss Flora of Mexico. Mem. N. Y. Bot. Gard. 69: 1-1113.

SIMPSON, B. 1979. A revision of the genus Polylepis (Rosaceae: Sanguisorbeae). Smithson. contrib. bot. 43: 1-62.

SIMPSON, B. 1986. Speciation and specialization of Polylepis in the Andes. In: VUILLEUMIER, F. \& M. Monasterio (eds.): High Altitude Tropical Biogeography. Pp. 304-316. Oxford Univ. Press.

TOIVONEN, J. M. 2014. Determinants of Polylepis (Rosaceae) forest distribution and treeline formation in the High Andes. Doctoral thesis. Department of Biology. Section of Biodiversity and Environmental Science. Painosalama Oy - Turku, Finland.

TRÓPICOS.org. Missouri Botanical Garden. Disponible en http://www.tropicos.org
VAN DER WIJK, R., MARGADANT, W. D. \& P. A. FLORSCHÜTZ. 1959. Index Muscorum. Volume 1 (A-C). Regnum Veg. 17: 548.

WOLF, J. H. D. 1993. Ecology of epiphytes and epiphyte communities in montane rain forest, Colombia. Tesis doctoral. Universidad de Amsterdam, Amsterdam.

ZANDER, R. H. 1993. Genera of the Pottiaceae: Mosses of harsh environments. Bull. Buffalo Soc. Nat. Sci 32: $1-278$.

ZUTTA, B., RUNDEL, P., SAATCHI, S., CASANA, J., GAUTHIER, P., SOTO, A., VELAZCO, Y. \& W. BUERMANN. 2012. Prediciendo la distribución de Polylepis: bosques Andinos vulnerables y cada vez más importantes. Rev. peru. biol 19 (2): 205-212.

Recibido el 1 de marzo de 2017, aceptado el 2 de junio de 2017. 
